\title{
CHALMERS
}

\section{Higher Order Beam Equations}

\author{
Master's Thesis in Solid and Fluid Mechanics
}

\section{HOSSEIN ABADIKHAH}

Department of Applied Mechanics

Division of Dynamics

CHALMERS UNIVERSITY OF TECHNOLOGY

Göteborg, Sweden 2011

Master's Thesis 2011:31 

MASTER'S THESIS 2011:31

\title{
Higher Order Beam Equations
}

\author{
Master's Thesis in Solid and Fluid Mechanics \\ HOSSEIN ABADIKHAH
}

Department of Applied Mechanics

Division of Dynamics

CHALMERS UNIVERSITY OF TECHNOLOGY

Göteborg, Sweden 2011 
Higher Order Beam Equations

HOSSEIN ABADIKHAH

(C)HOSSEIN ABADIKHAH, 2011

Master's Thesis 2011:31

ISSN 1652-8557

Department of Applied Mechanics

Division of Dynamics

Chalmers University of Technology

SE-412 96 Göteborg

Sweden

Telephone: + 46 (0)31-772 1000

Chalmers Reproservice

Göteborg, Sweden 2011 
Higher Order Beam Equations

Master's Thesis in Solid and Fluid Mechanics

HOSSEIN ABADIKHAH

Department of Applied Mechanics

Division of Dynamics

Chalmers University of Technology

\begin{abstract}
This thesis considers the analysis of a homogenous isotropic linearly elastic solid cylinder by assuming a displacement field that is a power series expansion in the radial coordinate. The solid cylinder is also referred to as a beam. Governing equations for the beam are obtained by inserting the power series ansatz into the equations of motion for linear elasticity, thereby obtaining recursion formulas which relates the coefficients of the power series with each other. Lateral boundary conditions on the beam's outer surface are expressed with the power series ansatz and the recursion formulas. The lateral boundary conditions form the basis of the governing equations. Dispersion relations and eigenfrequencies for the simply supported case are computed and compared to the exact theory, given by Pochhammer and Chree, and also with classical theories such as the Euler-Bernoulli and the Timoshenko theories. Displacement and stress fields are compared with the classical theories to show the deviancies of the proposed method.
\end{abstract}

Keywords: Wave propagation, Beam, Power series, Dispersion, Eigenfrequencies, Stress distribution 


\section{Contents}

$\begin{array}{ll}\text { Abstract } & \text { I }\end{array}$

$\begin{array}{lll}\text { Contents } & \text { III }\end{array}$

$\begin{array}{lll}\text { Preface } & \text { IV }\end{array}$

1 Introduction 1

2 Governing Equations 2

2.1 Equations of Motion . . . . . . . . . . . . . . . . 2

2.2 Stresses . . . . . . . . . . . . . . . . . . . . 2

2.3 Boundary Conditions . . . . . . . . . . . . . . . . . . . . 3

3 Power Series Ansatz 4

3.1 Recursion Formulas . . . . . . . . . . . . . . . . . . . 5

3.2 Expressions for Stresses . . . . . . . . . . . . . . . . . . . 6

3.3 Expressions for Boundary Conditions . . . . . . . . . . . . . . 7

3.4 The Wave Equation . . . . . . . . . . . . . . . . . . . . . . . . . 9

4 Dispersion Relations $\quad 10$

4.1 Beam Theory . . . . . . . . . . . . . . . . . . . . . 10

4.2 Exact Theory . . . . . . . . . . . . . . . . . . . . . . 10

4.3 Classical Theories . . . . . . . . . . . . . . . . . . . . . . 11

5 Dispersion curves $\quad 12$

5.1 Convergence study . . . . . . . . . . . . . . . . . . . . . . . . 12

6 Dispersion Relations for Torsion $\quad 18$

7 Eigenfrequencies $\quad 20$

7.1 Beam Theory . . . . . . . . . . . . . . . . . . 20

7.2 Exact Theory . . . . . . . . . . . . . . . . . . 20

7.3 Classical Theories . . . . . . . . . . . . . . . . . . . 20

7.4 Eigenfrequencies . . . . . . . . . . . . . . . . . . . 21

8 Mode Shapes and Stress Distributions 23

9 Concluding Remarks $\quad 27$

A Uncoupled Recursion Formulas 29

B Linear Differential Operators 30

C System of Beam Equations for $n=1 \quad 32$ 


\section{Preface}

This thesis studies whether it is possible to obtain governing equations for beams that approximate dispersion relations and eigenfrequencies better than classical theories such as the Euler-Bernoulli and Timoshenko theories with the assumption that the displacement fields can be written as power series ansatz. The work has been carried out from January 2011 to June 2011 at the Department of Applied Mechanics, Division of Dynamics Chalmers University of Technology, Sweden and with Dr. Peter Folkow as supervisor and examiner.

\section{Aknowledgements}

I would like to thank Dr. Peter Folkow for his guidance and support during this thesis.

Göteborg May 2011

Hossein Abadikhah 


\section{Introduction}

In the 19th century Pochhammer and later Chree independently laid the foundation of the exact theory regarding the elastodynamic behavior of homogenous isotropic linearly elastic cylinders. Specifically the modes of propagation for time harmonic waves were investigated. The investigated modes were categorized into three categories, longitudinal, circumferential and flexural modes. It was also found that there are infinite amounts of these types of modes and each mode had an infinite amount of eigenfrequencies associated with them. Before the exact theory was formulated another theory was used to analyze the behavior of flexural modes. This theory is named the Euler-Bernoulli beam theory and was conceived by Leonhard Euler and Daniel Bernoulli in the 18th century. The Euler-Bernoulli theory is based on an assumption for the displacement fields. If a cartesian coordinate system is chosen and the deflection of the beam is in the $z$-direction the assumption reads

$$
\begin{aligned}
& u_{x}(x, z, t)=-z \frac{\partial w}{\partial x}, \\
& u_{z}(x, z, t)=w(x, t),
\end{aligned}
$$

where $w$ is the transverse deflection of the midsurface. This assumption entails that plane cross sections of the beam remain plane after deformation and are perpendicular to the midsurface. This amounts to that the deformation is only caused by bending in the plane. During the beginning of the 20th century another theory regarding the behavior of beams was presented by Timoshenko. Timoshenko's theory introduced shear deformation and rotary inertia. Consequently plane cross sections remain plane after deformation but need not be perpendicular to the midsurface. The following assumptions were made for the Timoshenko theory in cartesian coordinates

$$
\begin{aligned}
& u_{x}(x, z, t)=-z \psi(x, t), \\
& u_{z}(x, z, t)=w(x, t) .
\end{aligned}
$$

As for the Euler-Bernoulli theory $w$ is the transverse deflection while $\psi$ is the rotation in plane around the $y$-axis in cartesian coordinates. In order to account for the variation of shear stress across the cross section Timoshenko introduced a shear coefficient that is dependent on material and geometry.

Another method for analyzing the behavior of elastic bodies and in particular rods was developed by Boström [2]. The method consists of choosing a proper power series ansatz for the displacement fields from which the governing equations can be obtained and consequently other relations such as stresses and dispersion equations. This method was used to investigate the governing equation and dispersion relations for rods [2]. The method was also used to investigate stresses and eigenfrequencies for finite rods [3]. Furthermore it was used also for cylindrical shells [4] and plates [5],[6].

The purpose of this thesis is to investigate whether the method described by [2] can be used to analyze beams. In order to examine if the attained governing equations yield acceptable results, the work presents dispersion relations, displacement and stress distributions and eigenfrequencies. 


\section{Governing Equations}

Regard a solid cylinder with radius $a$ and density $\rho$ described by cylindrical coordinates where the radial, circumferential and longitudinal coordinates are denoted by $r, \theta$ and $z$. The corresponding radial, circumferential and longitudinal displacement fields are denoted by $u, v$ and $w$. It is assumed that the material is homogenous, isotropic and linearly elastic with Lamé parameters $\lambda$ and $\mu$. The angle $\theta$ is measured from a vertical axis in a plane through the cross section of the cylinder as defined in [1]. Hereon the solid cylinder will be referred to as a beam.

\subsection{Equations of Motion}

The equations of motion, neglecting volume forces, in terms of stresses for a homogenous, isotropic and linearly elastic material expressed in cylindrical coordinates are written as [1]:

$$
\begin{aligned}
& \frac{\partial \sigma_{r r}}{\partial r}+\frac{1}{r} \frac{\partial \sigma_{r \theta}}{\partial \theta}+\frac{\partial \sigma_{r z}}{\partial z}+\frac{\sigma_{r r}-\sigma_{\theta \theta}}{r}=\rho \frac{\partial^{2} u}{\partial t^{2}} \\
& \frac{\partial \sigma_{r \theta}}{\partial r}+\frac{1}{r} \frac{\partial \sigma_{\theta \theta}}{\partial \theta}+\frac{\partial \sigma_{\theta z}}{\partial z}+2 \frac{\sigma_{r \theta}}{r}=\rho \frac{\partial^{2} v}{\partial t^{2}} \\
& \frac{\partial \sigma_{r z}}{\partial r}+\frac{1}{r} \frac{\partial \sigma_{\theta z}}{\partial \theta}+\frac{\partial \sigma_{z z}}{\partial z}+\frac{\sigma_{r z}}{r}=\rho \frac{\partial^{2} w}{\partial t^{2}} .
\end{aligned}
$$

\section{$2.2 \quad$ Stresses}

Stresses for a homogenous isotropic material can be written in terms of the displacement fields [4]. The relations are given as:

$$
\begin{aligned}
& \sigma_{r r}=\lambda\left[\frac{1}{r} \frac{\partial}{\partial r}(r u)+\frac{1}{r} \frac{\partial v}{\partial \theta}+\frac{\partial w}{\partial z}\right]+2 \mu \frac{\partial u}{\partial r}, \\
& \sigma_{\theta \theta}=\lambda\left[\frac{1}{r} \frac{\partial}{\partial r}(r u)+\frac{1}{r} \frac{\partial v}{\partial \theta}+\frac{\partial w}{\partial z}\right]+2 \mu\left[\frac{u}{r}+\frac{1}{r} \frac{\partial v}{\theta}\right], \\
& \sigma_{z z}=\lambda\left[\frac{1}{r} \frac{\partial}{\partial r}(r u)+\frac{1}{r} \frac{\partial v}{\partial \theta}+\frac{\partial w}{\partial z}\right]+2 \mu \frac{\partial w}{\partial z}, \\
& \sigma_{r \theta}=\mu\left[\frac{1}{r} \frac{\partial u}{\partial \theta}+\frac{\partial v}{\partial r}-\frac{v}{r}\right], \\
& \sigma_{r z}=\mu\left[\frac{\partial u}{\partial z}+\frac{\partial w}{\partial r}\right] \\
& \sigma_{\theta z}=\mu\left[\frac{\partial v}{\partial z}+\frac{1}{r} \frac{\partial w}{\partial \theta}\right] .
\end{aligned}
$$




\subsection{Boundary Conditions}

There are two types of boundary conditions, lateral conditions and end conditions. The lateral boundary conditions are applied along the beam's outer surface at $r=a$ and the end boundary conditions are applied on the end surfaces at $z=0, L$.

Furthermore there are two types of lateral boundary conditions, prescribed stresses or prescribed displacements and it is possible to have different fields prescribed on different parts of the outer surface. The following conditions can be assigned to a region of the surface

$$
\begin{array}{lll}
\sigma_{r r}(r=a)=t_{r} & \text { or } \quad u(r=a)=u_{r} \\
\sigma_{r \theta}(r=a)=t_{\theta} & \text { or } \quad v(r=a)=v_{\theta} \\
\sigma_{r z}(r=a)=t_{z} & \text { or } \quad w(r=a)=w_{z}
\end{array}
$$

The prescribed functions $t_{i}, u_{i}, v_{i}$ and $w_{i}$ where $i \in\{r, \theta, z\}$ are all known functions depending on $\theta, z$ and $t$. The end conditions are given by prescribing stresses or displacements that act at the end surfaces of the beam. Unlike the lateral conditions the entire end surface must be prescribed with one condition for each direction $(r, \theta, z)$. If different parts of the end surface have different conditions it is not possible to directly use the method presented here, instead one should use the theory discussed in [3]. However the method as presented here can be generalized to cases where conditions are dependent on $r, \theta$ and $t$. The combinations of end conditions are

$$
\begin{array}{lll}
\sigma_{r z}(z \in\{0, L\})=t_{r} & \text { or } \quad u(z \in\{0, L\})=u_{r}, \\
\sigma_{\theta z}(z \in\{0, L\})=t_{\theta} & \text { or } \quad v(z \in\{0, L\})=v_{\theta}, \\
\sigma_{z z}(z \in\{0, L\})=t_{z} & \text { or } \quad w(z \in\{0, L\})=w_{z} .
\end{array}
$$

The prescribed functions $t_{i}, u_{i}, v_{i}$ and $w_{i}$ where $i \in\{r, \theta, z\}$ are all known functions depending on $r, \theta$ and $t$. These functions are independent of the functions used for the lateral condition. 


\section{Power Series Ansatz}

It is assumed that the displacement fields can be expressed as a power series expansion in the radial coordinate. A Taylor series expansion of the exact theory is investigated in order to make the proper ansatz. It should also be noted that the circumferential dependence is assumed to be of trigonometric type in accordance with the exact theory [1].

For $m \in \mathbb{N}$ :

$$
\begin{array}{ll}
u=r^{m} \hat{u}^{(m)} \cos (m \theta), & \hat{u}^{(m)}=r^{-1} u_{m-1}^{(m)}+r u_{m+1}^{(m)}+r^{3} u_{m+3}^{(m)}+\ldots, \\
v=r^{m} \hat{v}^{(m)} \sin (m \theta), & v^{(m)}=r^{-1} v_{m-1}^{(m)}+r v_{m+1}^{(m)}+r^{3} v_{m+3}^{(m)}+\ldots, \\
w=r^{m} \hat{w}^{(m)} \cos (m \theta), & \hat{w}^{(m)}=w_{m}^{(m)}+r^{2} w_{m+2}^{(m)}+r^{4} w_{m+4}^{(m)}+\ldots
\end{array}
$$

For $m=0$ :

$$
\begin{aligned}
& u=\hat{u}^{(m)}, \\
& v=\hat{v}^{(m)}, \\
& w=\hat{w}^{(m)} .
\end{aligned}
$$

It is possible to deduce that the coefficients with indices that are smaller than $m-1$ for $u$ and $v$, and smaller than $m$ for $w$, vanish by expanding the exact theory (4.6) around $r=0$ or by assuming a complete ansatz where all indices larger than or equal to zero are included.

The solution type is determined by the parameter $m$. For instance if $m=1$ is chosen the solution will yield flexural waves. Similarly for $m=0$ the displacement fields $u$ and $w$ will generate longitudinal waves and the displacement field $v$ generates torsional waves. It should be noted that these two wave types are uncoupled. Furthermore the functions $u_{i}^{(m)}=u_{i}^{(m)}(z, t), v_{i}^{(m)}=v_{i}^{(m)}(z, t)$ and $w_{i}^{(m)}=w_{i}^{(m)}(z, t)$ for $i \in \mathbb{N}_{0}$ are smooth and differentiable. As noted before the displacement fields have the following properties by definition: $u_{i}^{(m)}=v_{i}^{(m)}=w_{i}^{(m)} \equiv 0$ for $i<0, u_{i}^{(m)}=v_{i}^{(m)} \equiv 0$ for $i<m-1$ and $w_{i}^{(m)} \equiv 0$ for $i<m$. The functions $u_{i}^{(m)}, v_{i}^{(m)}$ and $w_{i}^{(m)}$ are different functions for different values of $m$. From now on the superscript $(m)$ will be dropped. 


\subsection{Recursion Formulas}

By substituting the power series ansatz (3.1) into the expressions for stresses (2.2) and further substituting these into the the equations of motion (2.1) it is possible to obtain recursion formulas by identifying terms with equal powers of $r$ [2]. In the case of $m \geq 1$ two constraint equations are also obtained from the equations of motion. The recursion formulas are written as:

$$
\begin{aligned}
& {\left[(\lambda+2 \mu)(m+2 k+4)(m+2 k+2)-\mu m^{2}\right] u_{m+2 k+3}} \\
& +[(\lambda+\mu)(m+2 k+2) m-2 \mu m] v_{m+2 k+3} \\
& +(\lambda+\mu)(m+2 k+2) \frac{\partial w_{m+2 k+2}}{\partial z}+\mu \frac{\partial^{2} u_{m+2 k+1}}{\partial z^{2}}=\rho \frac{\partial^{2} u_{m+2 k+1}}{\partial t^{2}}, \quad \text { for } k \in \mathbb{N}_{0} \\
& -[(\lambda+\mu)(m+2 k+4) m+2 \mu m] u_{m+2 k+3} \\
& +\left[\mu(m+2 k+4)(m+2 k+2)-(\lambda+2 \mu) m^{2}\right] v_{m+2 k+3} \\
& -(\lambda+\mu) m \frac{\partial w_{m+2 k+2}}{\partial z}+\mu \frac{\partial^{2} v_{m+2 k+1}}{\partial z^{2}}=\rho \frac{\partial^{2} v_{m+2 k+1}}{\partial t^{2}}, \quad \text { for } k \in \mathbb{N}_{0} \\
& \mu\left[(m+2 k+2)^{2}-m^{2}\right] w_{m+2 k+2} \\
& +(\lambda+\mu)(m+2 k+2) \frac{\partial u_{m+2 k+1}}{\partial z}+(\lambda+\mu) m \frac{\partial v_{m+2 k+1}}{\partial z} \\
& +(\lambda+2 \mu) \frac{\partial^{2} w_{m+2 k}}{\partial z^{2}}=\rho \frac{\partial^{2} w_{m+2 k}}{\partial t^{2}}, \quad \text { for } k \in \mathbb{N}_{0}
\end{aligned}
$$

The constraint equations can be expressed as:

$$
\begin{aligned}
& u_{m-1}+v_{m-1}=0 \\
& {\left[(\lambda+2 \mu)(m+2) m-\mu m^{2}\right] u_{m+1}+\left[(\lambda+\mu) m^{2}-2 \mu m\right] v_{m+1}} \\
& +(\lambda+\mu) m \frac{\partial w_{m}}{\partial z}+\mu \frac{\partial^{2} u_{m-1}}{\partial z^{2}}=\rho \frac{\partial^{2} u_{m-1}}{\partial t^{2}}
\end{aligned}
$$

The constraint equations arise when the power series ansatz is inserted into the equations of motion (2.1). It is also possible to obtain the constraint (3.6) by substituting $k=-2$ in (3.3) or (3.4). The second constraint (3.7) can be obtained by setting $k=-1$ in (3.3). For the case of $m=0$ the constraint equations vanish in accordance with [2] and [3]. The recursion formulas are used to write all coefficients $u_{i}, v_{j}$ and $w_{k}$ as functions of the coefficient with lowest index. By studying the recursion formulas it is seen that the coefficients with the lowest indices are $u_{m+1}, v_{m+1}$ and $w_{m}$. But since the coefficients involved in the constraint equations have lower indices the resulting equations must be expressed in these coefficients. This creates several possibilities since it is possible to use $u_{m-1}$ or $u_{m+1}$ and $v_{m-1}$ or $v_{m+1}$. In this thesis all $u_{i}, v_{j}$ and $w_{k}$ where $i>m-1, j>m+1$ and $k>m$ are chosen to be written in terms of $u_{m-1}, v_{m+1}$, and $w_{m}$. For $m=0$ the functions $u_{i}, v_{j}$ and $w_{k}$ are expressed in $u_{1}, v_{1}$, and $w_{0}$ since the constraint equations vanish. The recursion equations $(3.3)$, (3.4) can be rewritten leading to an uncoupling between $u_{m+2 k+3}$ and $v_{m+2 k+3}$. In order to be brief these are presented in appendix A.

A more simple way to use the recursion formulas can be obtained by algebraically manipulating the equations presented in appendix A. It is then possible to write them in 
the following form

$$
\begin{aligned}
& u_{m+2 k+3}=d_{u u}(k, m) u_{m+2 k+1}+d_{u v}(k, m) v_{m+2 k+1}+d_{u w}(k, m) w_{m+2 k} \\
& v_{m+2 k+3}=d_{v u}(k, m) u_{m+2 k+1}+d_{v v}(k, m) v_{m+2 k+1}+d_{v w}(k, m) w_{m+2 k} \\
& w_{m+2 k+2}=d_{w u}(k, m) u_{m+2 k+1}+d_{w v}(k, m) v_{m+2 k+1}+d_{w w}(k, m) w_{m+2 k}
\end{aligned}
$$

where $d_{i j}$ are linear differential operators. The equation system can be written in matrix form

$$
\begin{aligned}
& \underbrace{\left[\begin{array}{c}
u_{m+2 k+3} \\
v_{m+2 k+3} \\
w_{m+2 k+2}
\end{array}\right]}_{\mathbf{u}_{k+1}}=\underbrace{\left[\begin{array}{lll}
d_{u u}(k, m) & d_{u v}(k, m) & d_{u w}(k, m) \\
d_{v u}(k, m) & d_{v v}(k, m) & d_{v w}(k, m) \\
d_{w u}(k, m) & d_{w v}(k, m) & d_{w w}(k, m)
\end{array}\right]}_{\mathbf{D}(k, m)} \underbrace{\left[\begin{array}{c}
u_{m+2 k+1} \\
v_{m+2 k+1} \\
w_{m+2 k}
\end{array}\right]}_{\mathbf{u}_{k}} \\
& \Leftrightarrow \mathbf{u}_{k+1}=\mathbf{D}(k, m) \mathbf{u}_{k}
\end{aligned}
$$

By using the recursion equation on matrix form (3.12) it is possible to express the displacement terms of $\mathbf{u}_{k+1}$ directly in $\mathbf{u}_{0}$ in the following way

$$
\mathbf{u}_{k+1}=\mathbf{A}(k, m) \mathbf{u}_{0}, \quad \text { where } \mathbf{A}(k, m)=\prod_{i=0}^{k} \mathbf{D}(k-i, m)
$$

The explicit expression for the different components of $\mathbf{D}$ are presented in appendix B.

\subsection{Expressions for Stresses}

As discussed in Section 3.1 expressions for stresses are obtained by substituting the power series ansatz (3.1) into the equations for stresses (2.2), which gives

$$
\begin{aligned}
\sigma_{r r} & =r^{m} \hat{\sigma}_{r r} \cos (m \theta), \\
\sigma_{\theta \theta} & =r^{m} \hat{\sigma}_{\theta \theta} \cos (m \theta), \\
\sigma_{z z} & =r^{m} \hat{\sigma}_{z z} \cos (m \theta), \\
\sigma_{r \theta} & =\mu r^{m} \hat{\sigma}_{r \theta} \sin (m \theta), \\
\sigma_{r z} & =\mu r^{m+1} \hat{\sigma}_{r z} \cos (m \theta), \\
\sigma_{\theta z} & =\mu r^{m+1} \hat{\sigma}_{\theta z} \sin (m \theta),
\end{aligned}
$$

where $\hat{\sigma}_{i j}=r^{-2} \hat{\sigma}_{i j, 0}+\hat{\sigma}_{i j, 1}+r^{2} \hat{\sigma}_{i j, 2}+\ldots+r^{2 k-2} \hat{\sigma}_{i j, k}+\ldots$

for the indices $i$ and $j$ being $\{r, \theta, z\}$. The stress terms $\hat{\sigma}_{i j, k}$ depend on $m$ and are generally different functions for different $m$. Subsequently each term can be written as

$$
\begin{aligned}
& \hat{\sigma}_{r r, k}=[(\lambda+2 \mu)(m+2 k)-2 \mu] u_{m+2 k-1}+\lambda m v_{m+2 k-1}+\lambda \frac{\partial w_{m+2 k-2}}{\partial z}, \\
& \hat{\sigma}_{\theta \theta, k}=[\lambda(m+2 k)+2 \mu] u_{m+2 k-1}+(\lambda+2 \mu) m v_{m+2 k-1}+\lambda \frac{\partial w_{m+2 k-2}}{\partial z}, \\
& \hat{\sigma}_{z z, k}=\lambda(m+2 k) u_{m+2 k-1}+\lambda m v_{m+2 k-1}+(\lambda+2 \mu) \frac{\partial w_{m+2 k-2}}{\partial z}, \\
& \hat{\sigma}_{r \theta, k}=(m+2 k-2) v_{m+2 k-1}-m u_{m+2 k-1}, \\
& \hat{\sigma}_{r z, k}=\frac{\partial u_{m+2 k-1}}{\partial z}+(m+2 k) w_{m+2 k}, \\
& \hat{\sigma}_{\theta z, k}=\frac{\partial v_{m+2 k-1}}{\partial z}-m w_{m+2 k},
\end{aligned}
$$

for $k \in \mathbb{N}_{0}$. 


\subsection{Expressions for Boundary Conditions}

By inserting the power series ansatz (3.1) and the stress series (3.14),(3.17) and (3.18) into the lateral boundary conditions explicit expressions can be obtained

$$
\begin{aligned}
& a^{m} \hat{\sigma}_{r r}(r=a) \cos (m \theta)=t_{r} \quad \text { or } \quad a^{m} \hat{u}(r=a) \cos (m \theta)=u_{r}, \\
& \mu a^{m} \hat{\sigma}_{r \theta}(r=a) \sin (m \theta)=t_{\theta} \quad \text { or } \quad a^{m} \hat{v}(r=a) \sin (m \theta)=v_{\theta}, \\
& \mu a^{m+1} \hat{\sigma}_{r z}(r=a) \cos (m \theta)=t_{z} \quad \text { or } \quad a^{m} \hat{w}(r=a) \cos (m \theta)=w_{z}, \\
& \text { for } \theta \in \theta^{*} \text { and } z \in z^{*},
\end{aligned}
$$

where $\theta^{*}$ and $z^{*}$ are a subregion of the outer surface. These boundary conditions constitute the beam equation from which the flexural motion of the beam are obtained. It should be noted that different regions of the beam are solved with different differential equations. For this thesis the outer surface is considered to be free resulting in the conditions that $\sigma_{r r}=\sigma_{r \theta}=\sigma_{r z} \equiv 0$ at $r=a$. By truncating these equations at different powers of $a$, beam equations of different order will be obtained. It is therefore important that the truncations are carried out so that sufficient amount of displacement terms are kept.

The stresses are truncated in such a way that the highest index for the longitudinal displacement term, $w_{i}$, is equal for the equations in the radial (3.26) and longitudinal (3.28) directions. The highest index for the longitudinal and circumferential displacement terms are equal for the equations in the radial (3.26) and circumferential (3.27) directions. This entails that if $\hat{\sigma}_{r z}(r=a)$ is truncated at $k=n$ involving terms up to $w_{m+2 n}$, then $\hat{\sigma}_{r r}(r=a)$ and $\hat{\sigma}_{r \theta}(r=a)$ are truncated both at $k=n+1$ involving terms up to $u_{m+2 n+1}$ and $v_{m+2 n+1}$. Another way to describe the truncation scheme is that if the longitudinal boundary condition is truncated at $a^{2 n-2}$ then the radial and circumferential boundary conditions will be truncated at $a^{2 n}$.

If the three boundary equations are reduced to a single beam equation, this truncation scheme produces a complete equation up to order $a^{2 n}$. However, it contains terms up to $a^{6 n}$, meaning that all $a^{2 n}$ terms will be generated but not all terms of higher power, rendering them incomplete. It should be noted that some algebraic manipulations are needed in order to write the reduced equation in a form where it is possible to distinguish terms of different orders. The derivatives in space and time for each order is increased by two. For instance the terms of order $a^{2 n}$ have derivatives of order $2 n+2$. The terms with highest derivative order are $6 n+2$ as the largest order terms for a truncation at $k=n$ are $a^{6 n}$. The system of beam equation obtained by the truncation $n=1$ is presented in appendix C.

If any boundary conditions on the displacements are given the above truncation scheme would also be used, i.e. the radial displacement and circumferential displacements would involve terms up to $u_{m+2 n+1}$ and $v_{m+2 n+1}$ while the longitudinal displacements would involve terms up to $w_{m+2 n}$. 
The discussed truncation scheme is only to be used when $m \geq 1$, while for $m=0$ the truncation scheme discussed in [3] should be used.

The only end boundary conditions that are investigated is the simply supported condition,

$$
\begin{aligned}
& u(z \in\{0, L\})=0, \\
& v(z \in\{0, L\})=0, \\
& \sigma_{z z}(z \in\{0, L\})=0 .
\end{aligned}
$$

This particular condition can be solved directly for the fixed frequency case by assuming a solution of the form

$$
\begin{aligned}
& u(z, t)=\sin \left(\frac{s \pi}{L} z\right) e^{-i \omega t} \\
& v(z, t)=\sin \left(\frac{s \pi}{L} z\right) e^{-i \omega t} \\
& w(z, t)=\cos \left(\frac{s \pi}{L} z\right) e^{-i \omega t}
\end{aligned}
$$

for $s \in \mathbb{N}$. 


\subsection{The Wave Equation}

By choosing $m=1$, yielding flexural waves, and truncating the stress series (3.26), (3.27) and (3.28) to the lowest order $n=1$ involving $u_{4}$ and $v_{4}$ for the radial and circumferential boundary conditions and involving $w_{3}$ for the longitudinal boundary condition, a system of equations are obtained that describe the flexural motion of the beam. The obtained system of equations can be expressed in $u_{0}, v_{2}$ and $w_{1}$ with the use of the recursion formulas and constraint equations. Furthermore it may be reduced to a single equation with the method used by [5]. The resulting equation can be expressed in any of the fields $u_{0}, v_{2}$ and $w_{1}$. The single equation contains all terms up to $a^{2}$ and some higher order terms which are truncated,

$$
\begin{aligned}
& \frac{\partial^{2} u_{0}}{\partial t^{2}}+\frac{a^{2} c_{T}^{2}}{24}\left(\alpha_{1} \frac{\partial^{4} u_{0}}{\partial z^{4}}-\frac{\alpha_{2}}{c_{T}^{2}} \frac{\partial^{4} u_{0}}{\partial z^{2} \partial t^{2}}+\frac{\alpha_{3}}{c_{T}^{4}} \frac{\partial^{4} u_{0}}{\partial t^{4}}\right)+\mathcal{O}\left(a^{4}\right)=0 \\
& c_{T}=\sqrt{\frac{\mu}{\rho}} \\
& \alpha_{1}=6 \frac{3 \lambda^{2}+8 \lambda \mu+4 \mu^{2}}{\lambda^{2}+3 \lambda \mu+2 \mu^{2}} \\
& \alpha_{2}=\frac{33 \lambda^{2}+95 \lambda \mu+58 \mu^{2}}{\lambda^{2}+3 \lambda \mu+2 \mu^{2}} \\
& \alpha_{3}=\frac{13 \lambda^{2}+43 \lambda \mu+32 \mu^{2}}{\lambda^{2}+3 \lambda \mu+2 \mu^{2}}
\end{aligned}
$$

If the truncation were not performed the resulting equation would be of order $a^{6}$ and would contain eighth derivatives in space and time. This equation cannot be solved directly by using the end boundary conditions presented in Section 2.3 since those conditions are expressed in all the displacement fields $u, v$ and $w$. Therefore the entire system of equations is needed to be able to find a solution.

All terms, even those that are truncated, are hyperbolic which is in accordance with [2]. Similarities between the Timoshenko beam equation (4.8) and the $a^{2}$ order wave equation can be found [1], both equations contain the same differential terms. If the term with mixed time and spatial derivative and the fourth order time derivative are neglected the Euler-Bernoulli beam equation (4.7) is obtained. This is seen as a verification of the analysis. 


\section{Dispersion Relations}

Validation of the analysis is done by comparing the dispersion curves using the current beam theory, the exact solution and classical beam theories such as the Euler-Bernoulli and the Timoshenko beam theories. When the expression "Beam theory" is used it refers to a choice of $m \geq 1$.

\subsection{Beam Theory}

Dispersion relations of a certain order can be obtained by truncating the boundary conditions stated in Section 3.3 and using the recursion formulas and constraint equations to write the equation system in terms of $u_{m-1}, v_{m+1}$ and $w_{m}$. The equations system is then reduced to a single equation [5]. It is important that the reduced equation is not truncated as is done in Section 3.4. If a truncation is done the reduced equation and the equation system obtained from the boundary conditions are not equivalent. Therefore all terms, even though some are incomplete, should be kept.

From the single differential equation the dispersion relation is obtained by assuming a solution that is harmonic both in space and time with a unit amplitude

$$
u_{m-1}=e^{i(k z-\omega t)} .
$$

Substituting this assumptions into the single differential equation results in a polynomial equation expressed in $k$ and $\omega$ from which the dispersion curves are obtained. It is also possible to express the dispersion relation directly from the equations system obtained by a truncation of the lateral boundary conditions without reduction to a single equation. In this case the three displacement fields $u_{m-1}, v_{m+1}$ and $w_{m}$ are assumed to be harmonic in space and time. By substituting this assumption into the equations system the dispersion relation is found when the coefficient matrix is singular. Both procedures give dispersion relations that are equivalent.

\subsection{Exact Theory}

The solution of the three dimensional elastodynamic equation in cylindrical coordinates can be expressed in four potentials [1]

$$
\begin{aligned}
& \phi=A J_{m}(p r) \cos (m \theta) e^{i(k z-\omega t)}, \\
& \psi_{z}=B J_{m}(q r) \sin (m \theta) e^{i(k z-\omega t)}, \\
& \psi_{r}=C J_{m+1}(q r) \sin (m \theta) e^{i(k z-\omega t)}, \\
& \psi_{\theta}=C J_{m+1}(q r) \cos (m \theta) e^{i(k z-\omega t)}, \\
& p=\sqrt{\frac{\omega^{2}}{c_{L}^{2}}-k^{2},} \\
& p=\sqrt{\frac{\omega^{2}}{c_{T}^{2}}-k^{2}}, \\
& c_{L}=\frac{\lambda+2 \mu}{\rho},
\end{aligned}
$$

where $J_{n}$ is the n:th Bessel function of first kind. $A, B$ and $C$ are constants that are determined by boundary conditions. The displacement fields can then be expressed in 
these four potentials [1]

$$
\begin{aligned}
u & =\frac{\partial \phi}{\partial r}+\frac{1}{r} \frac{\partial \psi_{z}}{\partial \theta}-\frac{\partial \psi_{\theta}}{\partial z}, \\
v & =\frac{1}{r} \frac{\partial \phi}{\partial \theta}+\frac{\partial \psi_{r}}{\partial z}-\frac{\partial \psi_{z}}{\partial r}, \\
w & =\frac{\partial \phi}{\partial z}+\frac{1}{r} \frac{\partial\left(r \psi_{\theta}\right)}{\partial r}-\frac{1}{r} \frac{\partial \psi_{r}}{\partial \theta} .
\end{aligned}
$$

Inserting the potentials into (4.5) gives $u, v$ and $w$ as a function of $r, \theta, z$ and $t$

$$
\begin{aligned}
& u=U(r) \cos (\theta) e^{i(k z-\omega t)}, \\
& v=V(r) \sin (\theta) e^{i(k z-\omega t)}, \\
& w=W(r) \cos (\theta) e^{i(k z-\omega t)}, \\
& \text { where } \\
& U(r)=A \frac{\partial}{\partial r} J_{m}(p r)+\frac{B}{r} J_{m}(q r)+i k C J_{m+1}(q r), \\
& V(r)=-\frac{A}{r} J_{m}(p r)-B \frac{\partial}{\partial r} J_{m}(q r)+i k C J_{m+1}(q r), \\
& W(r)=i k A J_{m}(p r)-\frac{C}{r} \frac{\partial}{\partial r}\left[r J_{m+1}(q r)\right]-\frac{C}{r} J_{m+1}(q r),
\end{aligned}
$$

The boundary condition for the beam are defined in Section 3.3, namely that $\sigma_{r r}=$ $\sigma_{r \theta}=\sigma_{r z} \equiv 0$ at $r=a$. The displacements (4.5) can then be inserted into the expressions for $\sigma_{r r}, \sigma_{r \theta}$ and $\sigma_{r z}(2.2)$ resulting in three equations from which the dispersion relation is obtained by eliminating the constants $A, B$ and $C$.

\subsection{Classical Theories}

It is straightforward to obtain dispersion relations for the classical beam theories, such as the Euler-Bernoulli and the Timoshenko beam theories. The differential equation for the Euler-Bernoulli beam theory and the Timoshenko beam theory are

$$
\begin{aligned}
& \frac{\partial^{2} u_{E B}}{\partial t^{2}}+\frac{E I}{A \rho} \frac{\partial^{4} u_{E B}}{\partial z^{4}}=0, \\
& \frac{\partial^{2} u_{T}}{\partial t^{2}}+\frac{E I}{A \rho} \frac{\partial^{4} u_{T}}{\partial z^{4}}-\frac{1}{A}\left(I+\frac{E I}{G \kappa}\right) \frac{\partial^{4} u_{T}}{\partial z^{2} \partial t^{2}}+\frac{I \rho}{G \kappa^{2} A} \frac{\partial^{4} u_{T}}{\partial t^{4}}=0,
\end{aligned}
$$

where $\rho$ is the density, $A$ is the cross sectional area, $E$ is the elastic modulus, $G$ is the shear modulus, $I$ is the area moment of inertia and $\kappa$ is the shear coefficient. The shear coefficient is chosen to be $\kappa=\frac{6(1+\nu)^{2}}{\left(7+12 \nu+4 \nu^{2}\right)}$ [7] where $\nu$ is Poisson's ratio. Observe that these equations are written in a cartesian coordinate system where the $z$-axis is along the beam and the deflection is in the $y$-direction. The dispersion relations are then obtained by assuming that the displacement field $u=u(z, t)$ is harmonic in time and space with a unit amplitude, i.e. $u=e^{i(k z-\omega t)}$ for both Euler-Bernoulli and Timoshenko theories. 


\section{Dispersion curves}

Two types of results for the dispersion relations are presented, the first being a convergence study where the current beam theory is compared to the exact solution for different truncations and two different solution types namely $m=1$ and $m=2$. Secondly a comparison between the exact solution, the classical theories and the truncated wave equation (3.31) is conducted.

\subsection{Convergence study}

The convergence comparison is made between the three first dispersion curves obtained from the exact solution and the current beam theory for truncations at $n=1,2,3,4$. Hence the comparison is made between the exact theory and the $a^{6}, a^{12}, a^{18}, a^{24}$ order theories. This comparison is done for $m=1$ and $m=2$. The dispersion relations have generally complex solutions but for this comparison only the real solutions are considered. Furthermore not all curves will be generated by the different truncations. Only those curves that are generated will be compared against the exact solution. This will result in that certain truncations do not show three curves in the comparison.

The dispersion curves figures 5.1-5.4 are all of the type $m=1$, while figures 5.5-5.8 are all of type $m=2$.

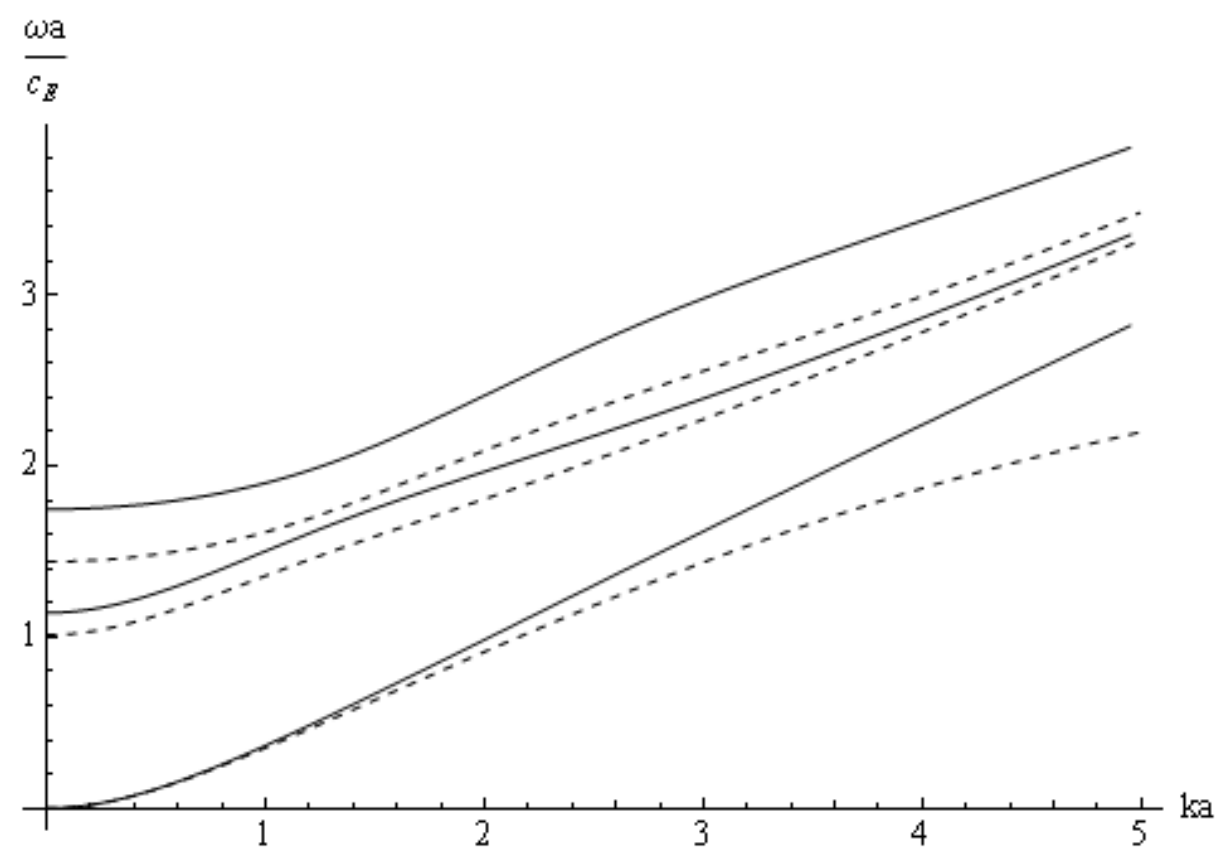

Figure 5.1: Solution to the dispersion relation for $m=1$ : Exact -,

Truncation at $n=1---$ 


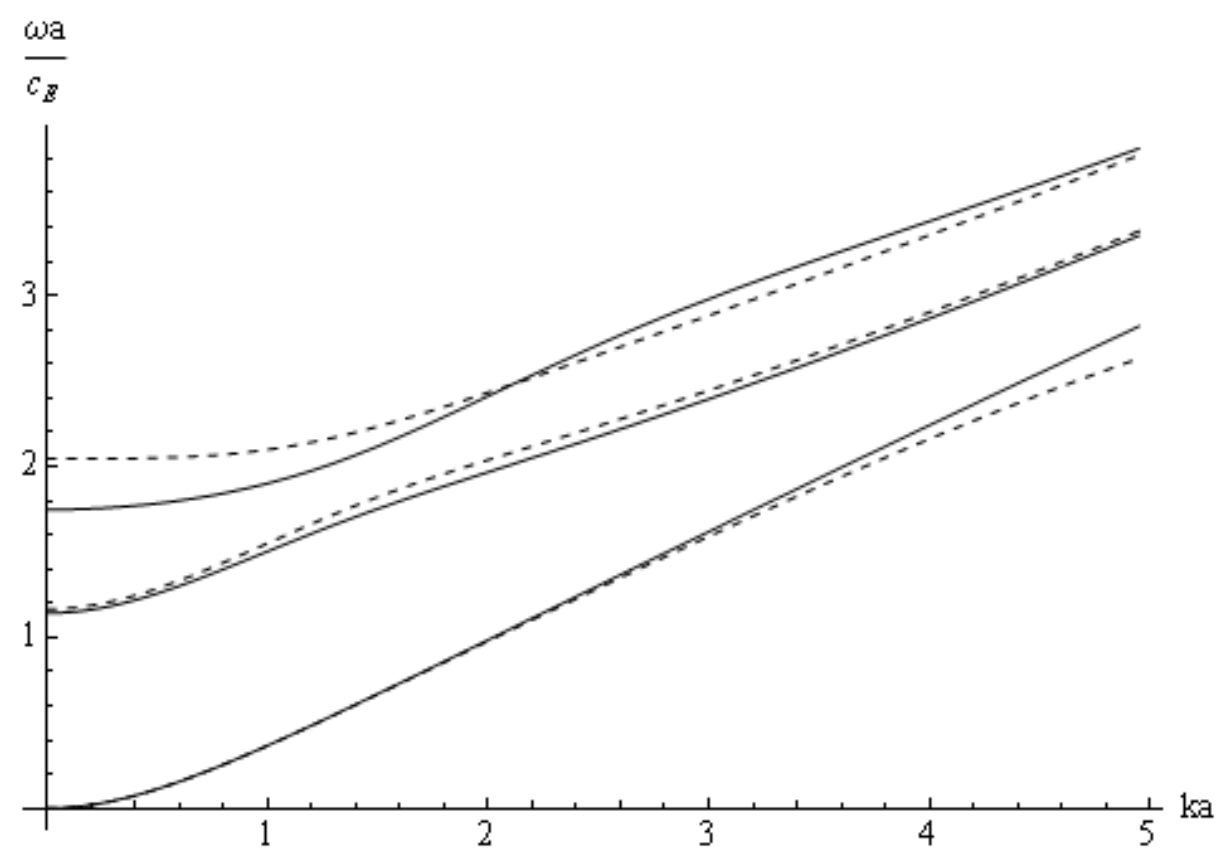

Figure 5.2: Solution to the dispersion relation for $m=1$ : Exact Truncation at $n=2---$

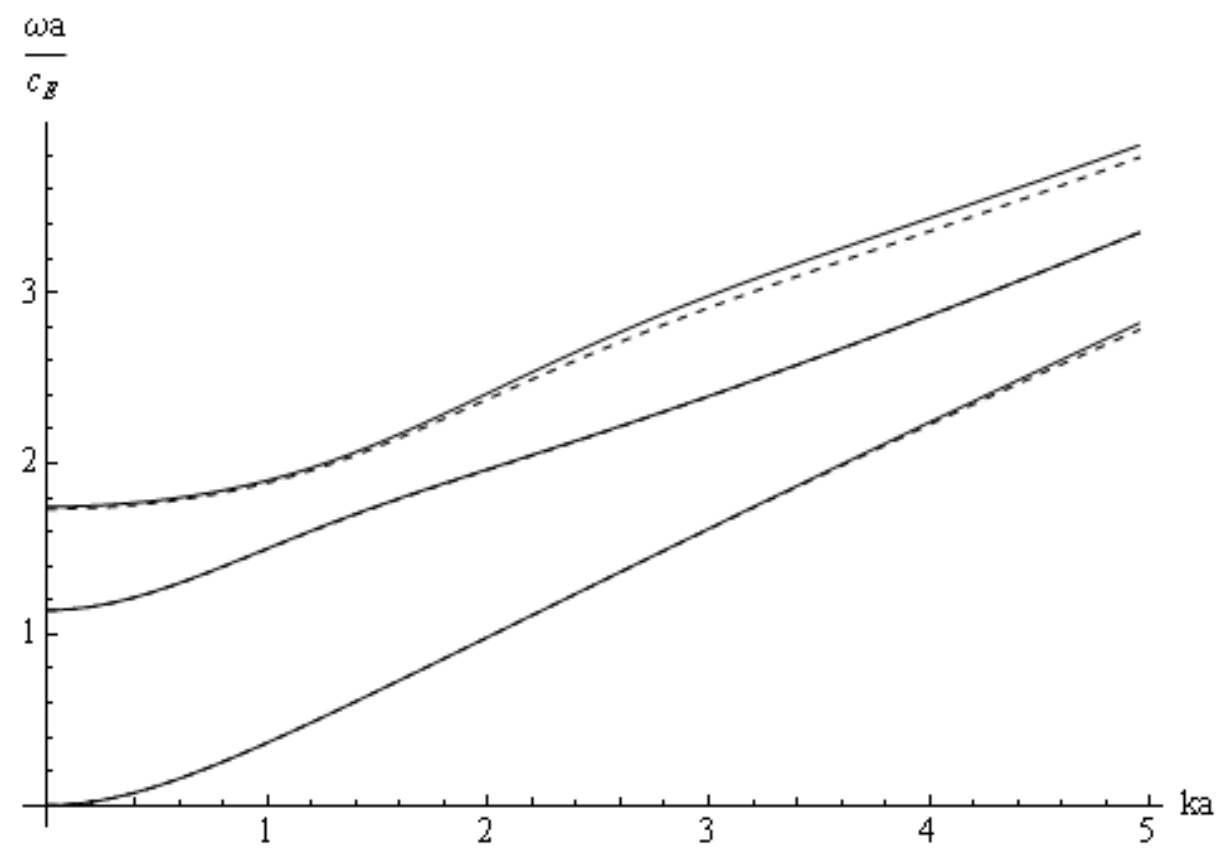

Figure 5.3: Solution to the dispersion relation for $m=1$ : Exact Truncation at $n=3---$ 


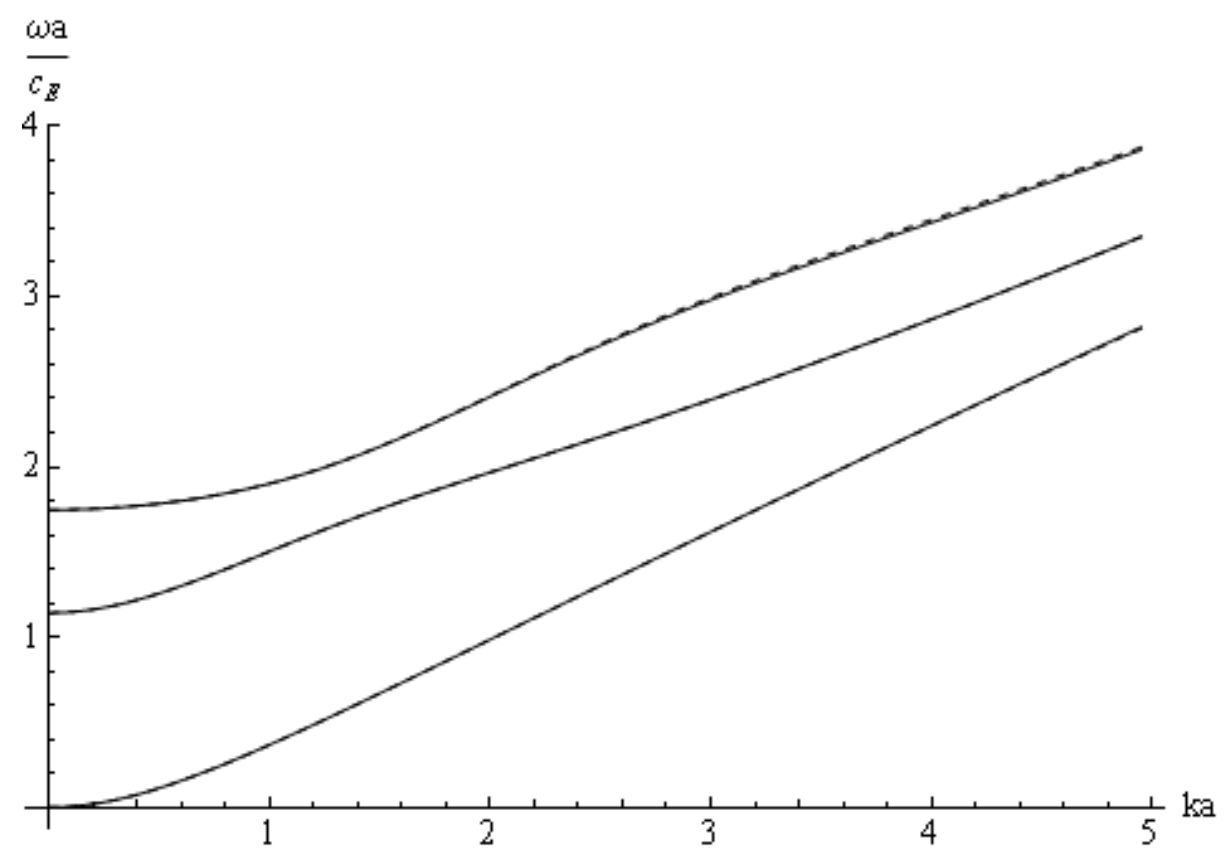

Figure 5.4: Solution to the dispersion relation for $m=1$ : Exact -, Truncation at $n=4---$

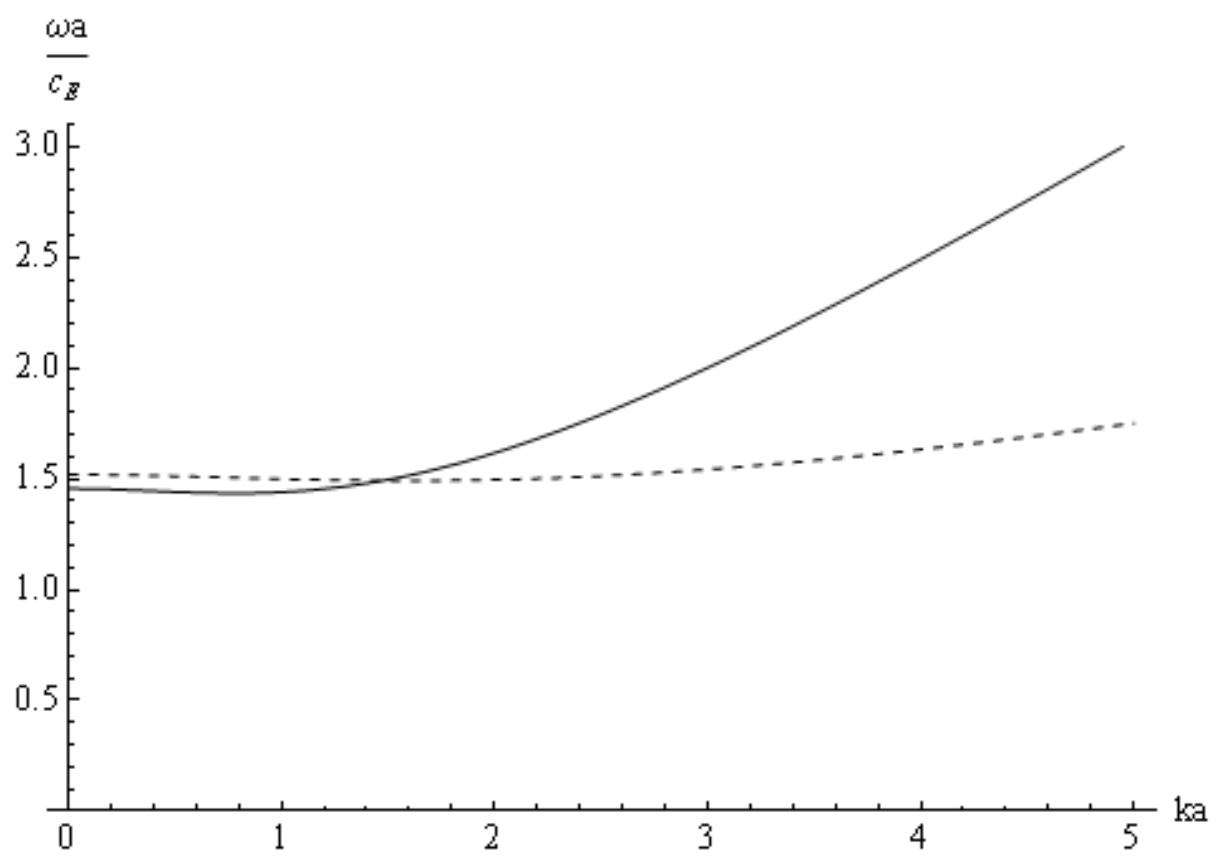

Figure 5.5: Solution to the dispersion relation for $m=2$ : Exact Truncation at $n=1---$ 


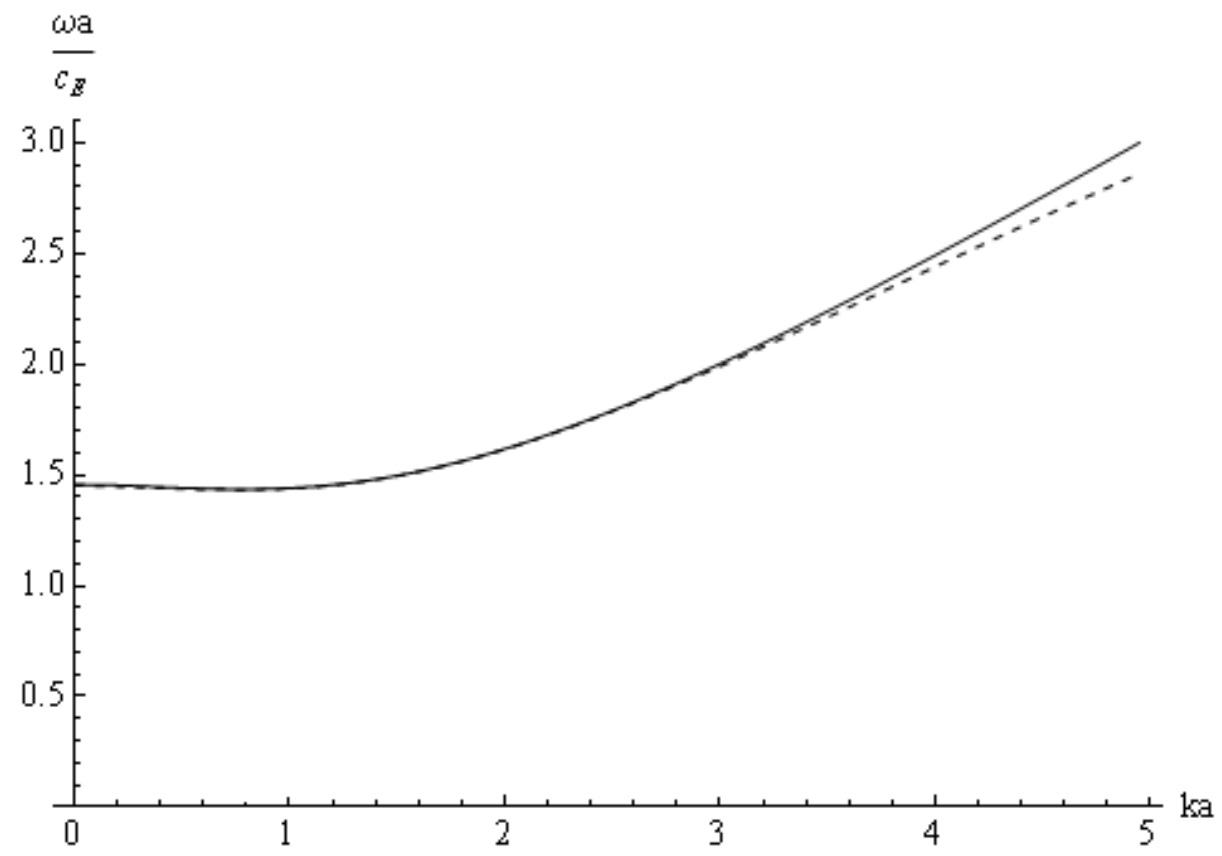

Figure 5.6: Solution to the dispersion relation for $m=2$ : Exact Truncation at $n=2---$

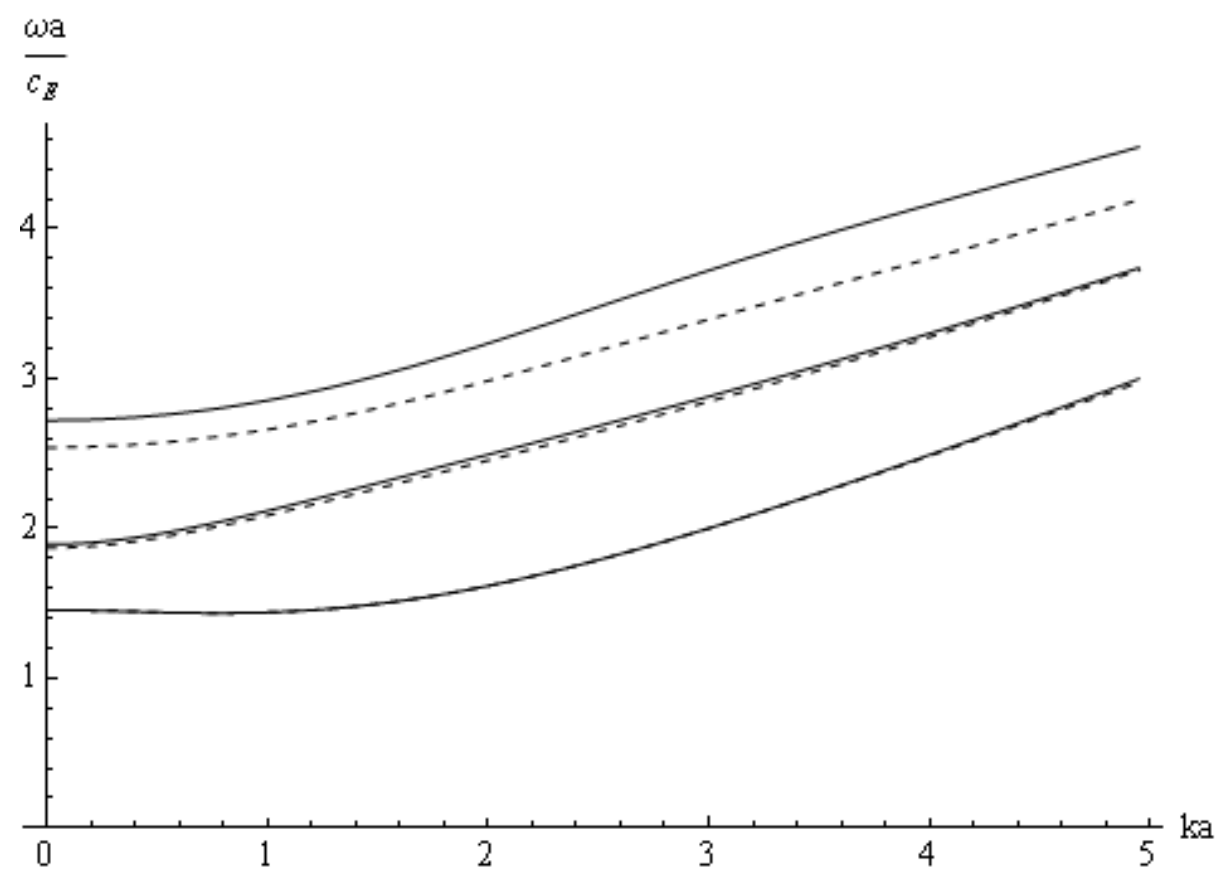

Figure 5.7: Solution to the dispersion relation for $m=2$ : Exact -, Truncation at $n=3---$ 


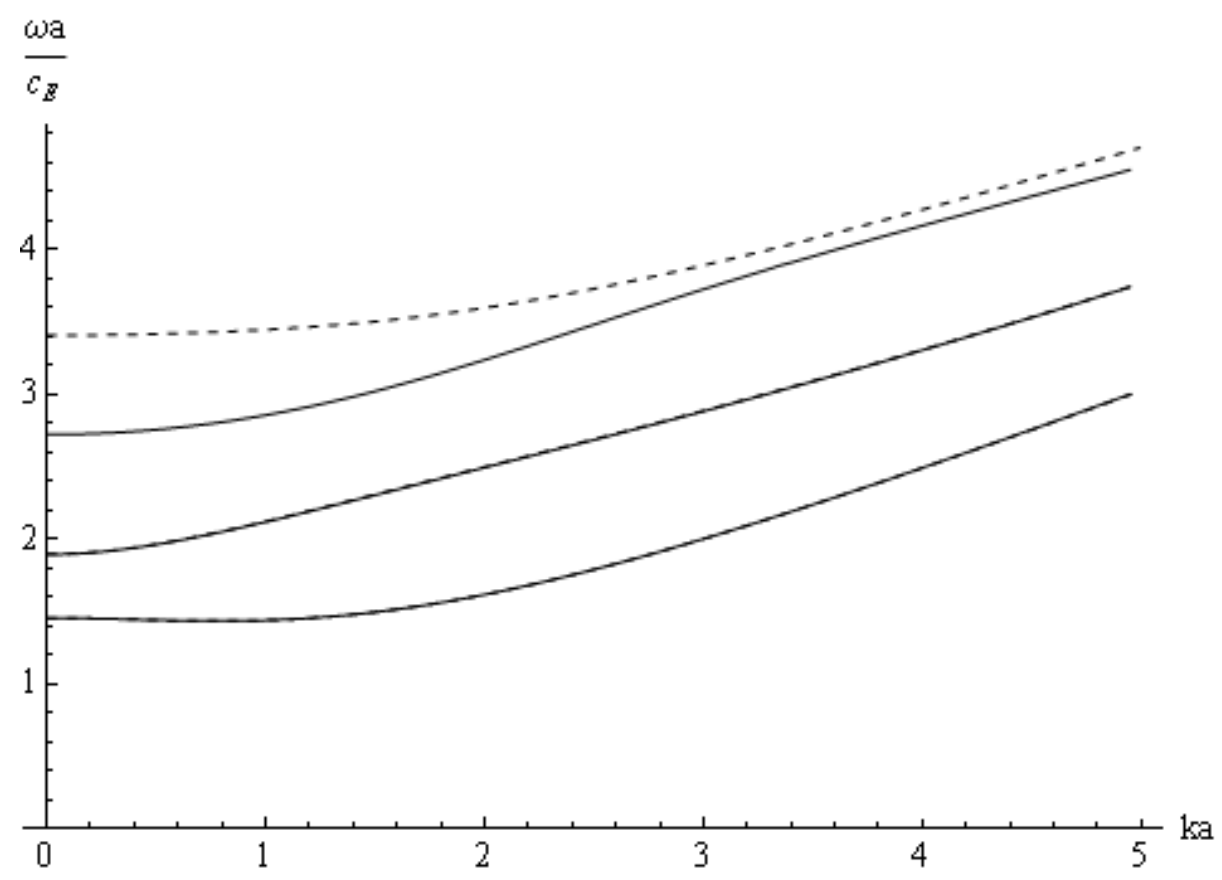

Figure 5.8: Solution to the dispersion relation for $m=2$ : Exact -, Truncation at $n=4---$

By studying the dispersion curves it is seen that a higher truncation order gives a more accurate theory. The third curve for both cases is the least accurate, this could be accommodated by increasing the truncation order. The behavior for higher modes which start at a higher cut of frequency are approximated worse for a specific truncation, which is characteristic of this beam theory. Also the theory for $m=2$ is generally less accurate than for $m=1$. 
The comparison between the classical theories, exact solution and the $a^{2}$ order wave equation is treated in figure 5.9.

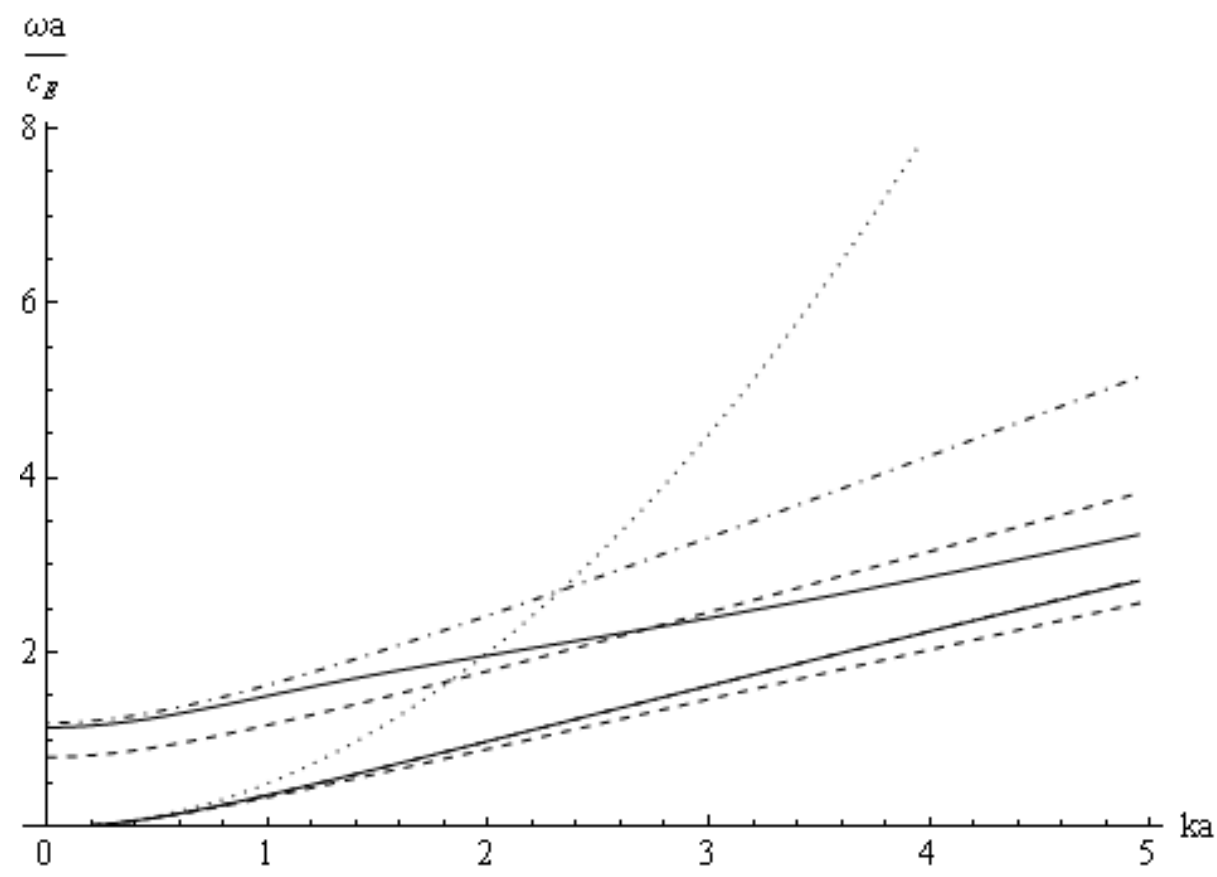

Figure 5.9: Solution to the dispersion relation: Exact,$- a^{2}---$, Timoshenko $-\cdot-$, Euler-Bernoulli ...

As expected the Euler-Bernoulli beam theory is only acceptable at small wave numbers and it progressively deteriorates as the wave number is increased. The $a^{2}$ order beam theory approximates the first dispersion curve well for small wave numbers but the approximation deteriorates as the wave number is increased. The second dispersion curve is approximated less accurately particulary at small wave numbers. The Timoshenko beam theory approximates the first dispersion curve very accurately and approximates the second curve fairly well at small wave numbers but the approximation deteriorates as the wave number increases. 


\section{Dispersion Relations for Torsion}

For the particular case of $m=0$ the theory results in longitudinal and torsional modes. These two mode types are also uncoupled. This uncoupling makes it possible to derive dispersion relations for the torsional modes analytically. The case of longitudinal waves is treated in [2],[3]. For the analysis of the torsional dispersion relation the displacement fields $u$ and $w$ are considered to be nonexistent. It is assumed that the outer surface of the cylinder is free resulting in the boundary condition

$$
\sigma_{r \theta}(r=a)=0
$$

By using the expression (3.17) it is possible to write the boundary condition in the following manner

$$
\sum_{k=0}^{n-1}(k+1) v_{2 k+3} a^{2 k+2}+\mathcal{O}\left(a^{2 n+2}\right)=0 .
$$

The recursion formula (3.4) can be simplified to

$$
v_{2 k+3}=\frac{1}{4(k+1)(k+2)}\left(\frac{1}{c_{T}^{2}} \frac{\partial^{2}}{\partial t^{2}}-\frac{\partial^{2}}{\partial z^{2}}\right) v_{2 k+1} \quad \text { for } k=0,1,2, \ldots
$$

With use of (3.13) the recursion formula can be written in terms of $v_{1}(z, t)$

$$
v_{2 k+3}=\frac{1}{4^{k+1}(k+1) !(k+2) !}\left(\frac{1}{c_{T}^{2}} \frac{\partial^{2}}{\partial t^{2}}-\frac{\partial^{2}}{\partial z^{2}}\right)^{k+1} v_{1}(z, t) .
$$

Inserting (6.4) into the boundary condition (6.2) and algebraically manipulating the sum the following expression is obtained up to infinite order

$$
-\sum_{k=0}^{\infty} \frac{(-1)^{k}}{k !(k+2) !}\left(\frac{a}{2} \sqrt{\frac{\partial^{2}}{\partial z^{2}}-\frac{1}{c_{T}^{2}} \frac{\partial^{2}}{\partial t^{2}}}\right)^{2 k+2} v_{1}(z, t)=0
$$

By assuming a harmonic wave in both time and space $v_{1}(z, t)=e^{i\left(k_{z} z-\omega t\right)}$ which is inserted into (6.5) the dispersion relation is obtained by some manipulations

$$
\begin{aligned}
& -\sum_{k=0}^{\infty} \frac{(-1)^{k}}{k !(k+2) !}\left(\frac{1}{2} q a\right)^{2 k+2}=0, \\
& \Leftrightarrow \quad-J_{2}(q a)=0,
\end{aligned}
$$

where $q=\sqrt{\frac{\omega^{2}}{c_{T}^{2}}-k_{z}^{2}}$ and $J_{2}$ is the second Bessel function.

Equation (6.7) may be extended to

$$
-(q a) J_{2}(q a)=0
$$


From [8] a recursion formula for cylindrical Bessel functions can be found

$J_{p-1}(x)+J_{p+1}(x)=\frac{2 p}{x} J_{p}(x)$,

and by setting $p=1$ a relation involving $J_{2}$ is obtained

$J_{0}(x)+J_{2}(x)=\frac{2}{x} J_{1}(x)$,

$\Rightarrow-x J_{2}(x)=x J_{0}(x)-2 J_{1}(x)$.

Denoting $x=q a$ gives

$$
-(q a) J_{2}(q a)=(q a) J_{0}(q a)-2 J_{1}(q a)=0 .
$$

The dispersion relation (6.9) is the exact dispersion relation for torsional modes given by [1]. 


\section{Eigenfrequencies}

A comparison study of the eigenfrequencies for the simply supported case is conducted for $m=1$ with the current beam theory, the exact theory and the classical theories. Two different beam configurations are considered with the ratios $L / a=10$ and $L / a=2$.

\subsection{Beam Theory}

As with the dispersion relations the frequency equation is obtained by truncating the boundary conditions in Section 3.3 and using the recursion formulas and constraint equations to express the equation system in the displacement fields $u_{m-1}, v_{m+1}$ and $w_{m}$. Furthermore the displacement fields, $u_{m-1}, v_{m+1}$ and $w_{m}$, are assumed to be of trigonometric type as stated in (3.30). The eigenfrequencies can then be obtained by letting the obtained coefficient matrix be singular.

\subsection{Exact Theory}

The exact eigenfrequencies for the simply supported boundary conditions are acquired by rewriting the potentials (4.1) to (4.4) in the following manner

$$
\begin{aligned}
& \phi=A J_{m}(p r) \cos (m \theta) \sin \left(\frac{s \pi}{L} z\right) e^{-i \omega t}, \\
& \psi_{z}=B J_{m}(q r) \sin (m \theta) \sin \left(\frac{s \pi}{L} z\right) e^{-i \omega t}, \\
& \psi_{r}=C J_{m+1}(q r) \sin (m \theta) \cos \left(\frac{s \pi}{L} z\right) e^{-i \omega t}, \\
& \psi_{\theta}=C J_{m+1}(q r) \cos (m \theta) \cos \left(\frac{s \pi}{L} z\right) e^{-i \omega t}, \quad \text { for } s=1,2, \ldots
\end{aligned}
$$

Using (4.5) the displacement fields are acquired, which are then inserted into the equations for stresses (2.2). The stresses are then inserted into the lateral boundary conditions at the resulting coefficient matrix is set to be singular. An equation is obtained from which the eigenfrequencies are calculated.

\subsection{Classical Theories}

The frequency equations for the classical beam theories, Euler-Bernoulli and Timoshenko beam theory, are obtained by assuming a displacement field of trigonometric type. From this ansatz a equation in the frequency $\omega$ is obtained from which the eigenfrequencies are calculated. The ansatz for the Euler-Bernoulli theory is

$$
u_{E B}=A \sin \left(\frac{s \pi}{L} z\right) e^{-i \omega t}
$$

and for the Timoshenko theory the ansatz is

$$
\begin{aligned}
& u_{T}=A \sin \left(\frac{s \pi}{L} z\right) e^{-i \omega t} \\
& \psi=B \cos \left(\frac{s \pi}{L} z\right) e^{-i \omega t} .
\end{aligned}
$$




\subsection{Eigenfrequencies}

The eigenfrequencies are computed for the following material parameters $E=210$ GPa, $\nu=0.3, \rho=7800 \mathrm{~kg} / \mathrm{m}^{3}$ and $L=1$ meter. For each $s$ in (3.30) there are infinitely many eigenfrequencies. In this comparison study only the first two of these frequencies are considered. This means that for each truncation ten eigenfrequencies are computed for $s=1,2, \ldots, 5$. The Euler-Bernoulli theory can only generate one eigenfrequency for each $s$ while the Timoshenko theory generates two eigenfrequencies. The current beam theory generates $3 n+1$ eigenfrequencies for each $s$ where $n$ is the truncation order.

\begin{tabular}{l|ccccc} 
Theory & $\Omega_{1}$ & $\Omega_{2}$ & $\Omega_{3}$ & $\Omega_{4}$ & $\Omega_{5}$ \\
\hline Exact & 0.0472148 & 0.169554 & 0.334223 & 0.519392 & 0.713910 \\
$n=1$ & 0.0469096 & 0.166193 & 0.323078 & 0.495735 & 0.673345 \\
$n=2$ & 0.0472135 & 0.169502 & 0.333877 & 0.518233 & 0.711147 \\
$n=3$ & 0.0472148 & 0.169553 & 0.334218 & 0.519362 & 0.713805 \\
$n=4$ & 0.0472148 & 0.169554 & 0.334223 & 0.519392 & 0.713908 \\
Timoshenko & 0.0472138 & 0.169520 & 0.334044 & 0.518933 & 0.713098 \\
Euler-Bernoulli & 0.0493480 & 0.197392 & 0.444132 & 0.789568 & 1.23370
\end{tabular}

Table 7.1: The first eigenfrequencies $\Omega=\omega a / c_{E}$ for each $s$ and $L / a=10$

\begin{tabular}{l|ccccc} 
Theory & $\Omega_{1}$ & $\Omega_{2}$ & $\Omega_{3}$ & $\Omega_{4}$ & $\Omega_{5}$ \\
\hline Exact & 0.713910 & 1.70790 & 2.67650 & 3.61407 & 4.52813 \\
$n=1$ & 0.673345 & 1.50529 & 2.11342 & 2.52077 & 2.85535 \\
$n=2$ & 0.711147 & 1.67482 & 2.52268 & 3.09726 & 3.40839 \\
$n=3$ & 0.713805 & 1.70396 & 2.64651 & 3.47912 & 4.06835 \\
$n=4$ & 0.713908 & 1.70758 & 2.67165 & 3.58324 & 4.40517 \\
Timoshenko & 0.713098 & 1.70743 & 2.68640 & 3.65006 & 4.60482 \\
Euler-Bernoulli & 1.23370 & 4.93480 & 11.1033 & 19.7392 & 30.8425
\end{tabular}

Table 7.2: The first eigenfrequencies $\Omega=\omega a / c_{E}$ for each $s$ and and $L / a=2$

\begin{tabular}{l|ccccc} 
Theory & $\Omega_{1}$ & $\Omega_{2}$ & $\Omega_{3}$ & $\Omega_{4}$ & $\Omega_{5}$ \\
\hline Exact & 1.19007 & 1.31342 & 1.47264 & 1.63547 & 1.78408 \\
$n=1$ & 1.06057 & 1.18027 & 1.33004 & 1.47976 & 1.61995 \\
$n=2$ & 1.21612 & 1.34605 & 1.51705 & 1.69516 & 1.85505 \\
$n=3$ & 1.18875 & 1.31163 & 1.47005 & 1.63198 & 1.78005 \\
$n=4$ & 1.19012 & 1.31350 & 1.47277 & 1.63566 & 1.78431 \\
Timoshenko & 1.24697 & 1.38920 & 1.58623 & 1.81525 & 2.06404
\end{tabular}

Table 7.3: The second eigenfrequencies $\Omega=\omega a / c_{E}$ for each $s$ and $L / a=10$ 


\begin{tabular}{l|ccccc} 
Theory & $\Omega_{1}$ & $\Omega_{2}$ & $\Omega_{3}$ & $\Omega_{4}$ & $\Omega_{5}$ \\
\hline Exact & 1.78408 & 2.45840 & 3.22656 & 4.07576 & 4.97841 \\
$n=1$ & 1.61995 & 2.34328 & 3.16277 & 4.04780 & 4.97633 \\
$n=2$ & 1.85505 & 2.50659 & 3.25513 & 4.09022 & 4.98908 \\
$n=3$ & 1.78005 & 2.45594 & 3.22596 & 4.07526 & 4.97888 \\
$n=4$ & 1.78431 & 2.45854 & 3.22668 & 4.07550 & 4.97794 \\
Timoshenko & 2.06404 & 3.44814 & 4.93105 & 6.45188 & 7.99087
\end{tabular}

Table 7.4: The second eigenfrequencies $\Omega=\omega a / c_{E}$ for each $s$ and $L / a=2$

Firstly, the second eigenfrequency for a particular $s$ cannot be computed with the EulerBernoulli beam theory. Furthermore it is seen that the current beam theory is a good approximation of the eigenfrequencies as more terms are considered i.e. it is truncated at a larger number. Two observations should be noted, firstly the approximate eigenfrequency deteriorates as $s$ increases and secondly the approximation deteriorates as the ratio $L / a$ decreases. That the approximation deteriorates as $L / a$ decreases is natural since the beam like structure is lost. In order to overcome both these problems the truncation order must be increased. The Timoshenko beam theory approximates the exact eigenfrequencies well at large ratios of $L / a$ and for the first eigenfrequencies for a particular $s$. But the approximation deteriorates as the ratio $L / a$ is decreased, when $s$ is increased and for the second eigenfrequency for a particular $s$. The Euler-Bernoulli is only a good approximation for large $L / a$. By studying the tables it is seen that the current beam theory is a good method for approximating eigenfrequencies. 


\section{Mode Shapes and Stress Distributions}

In order to illustrate the differences of the current beam theory and the classical theories, modes shapes and stress distributions for several cases are compared for $m=1$. Although not all possible mode shapes or stress distributions are presented several interesting cases that show the general tendencies are studied. All the displacement fields and stresses are transformed to a cylindrical coordinate system. In all the studied cases $\theta$ is chosen so that the trigonometric dependencies is unity. The cases studied are simply supported with $L / a=10$ for the lowest eigenfrequency in the case of each theory. The current beam theory uses $n=4$ truncation since the difference between this theory and exact is small only the $n=4$ is plotted. Although $n=1$ may also be considered the difference between $n=4$ and $n=1$ theories is so small that only the $n=4$ theory is chosen. The radial displacement $u$ computed as a function of the radius for $z=3 L / 4$ is presented in figure 8.1.

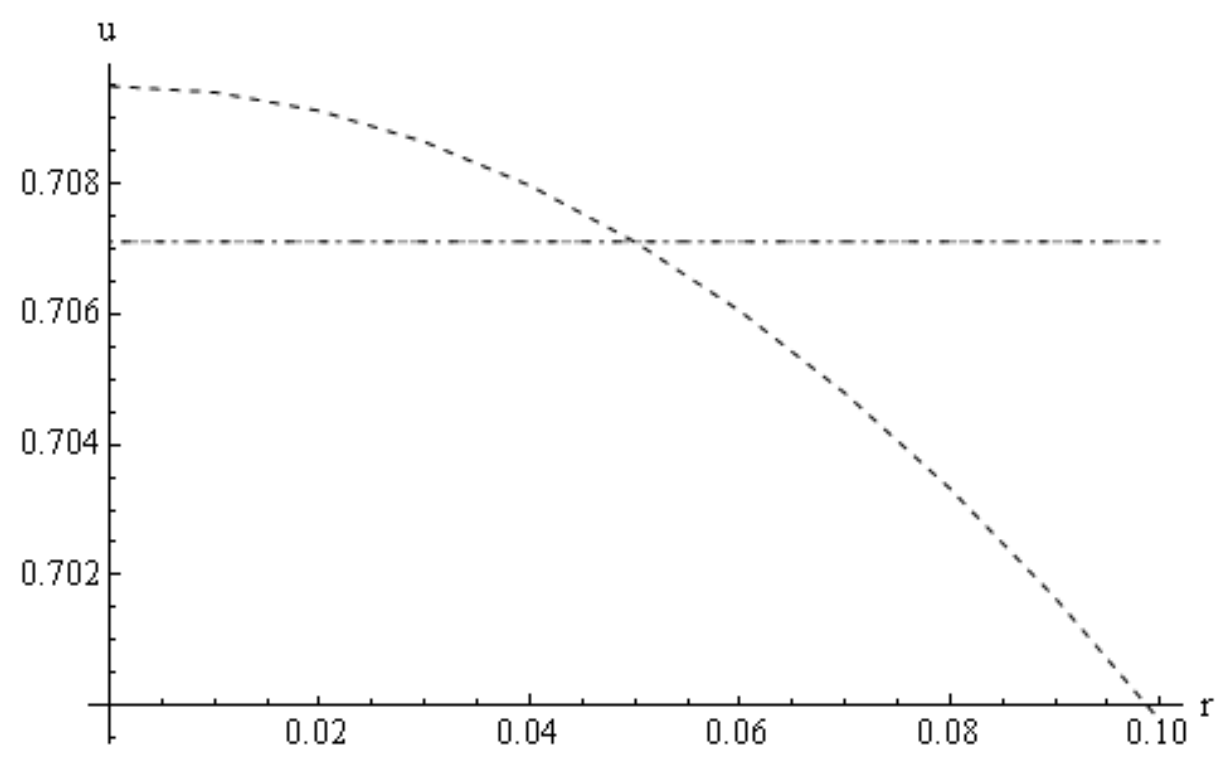

Figure 8.1: $u(r, z=3 L / 4)$ : Truncation at $n=4---$, Timoshenko $-\cdot-$, Euler-Bernoulli ...

The current beam theory describes a radial displacement that varies while both the Euler-Bernoulli and the Timoshenko theories describe a constant displacement field lying on top of each other. The circumferential displacement field behaves in a similar fashion.

In figure 8.2 the stress $\sigma_{z z}$ is plotted as a function of the lateral coordinate $z$ for $r=a$ i.e. the outer surface of the beam. 


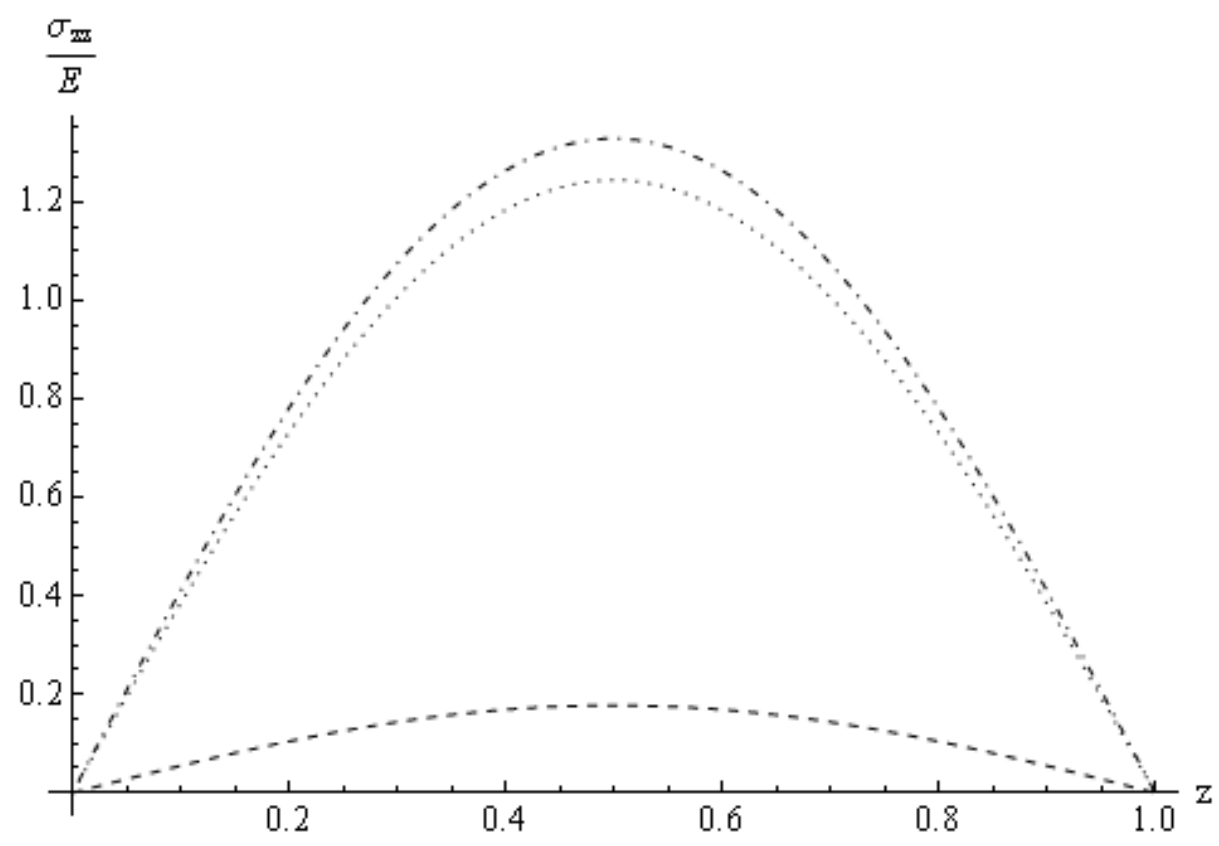

Figure 8.2: $\sigma_{z z}(r=a, z)$ : Truncation at $n=4---$, Timoshenko $-\cdot-$, Euler-Bernoulli $\cdots$

The behavior of $\sigma_{z z}$ is also characteristic for $\sigma_{r r}$ and $\sigma_{\theta \theta}$ when they are computed for a particular radius. In all cases the classical theories produces larger values than the current beam theory. It should also be noted that that this stress is at the outer surface of the beam.

The shear stress $\sigma_{\theta z}$ is also computed for $r=a$, presented in figure 8.3

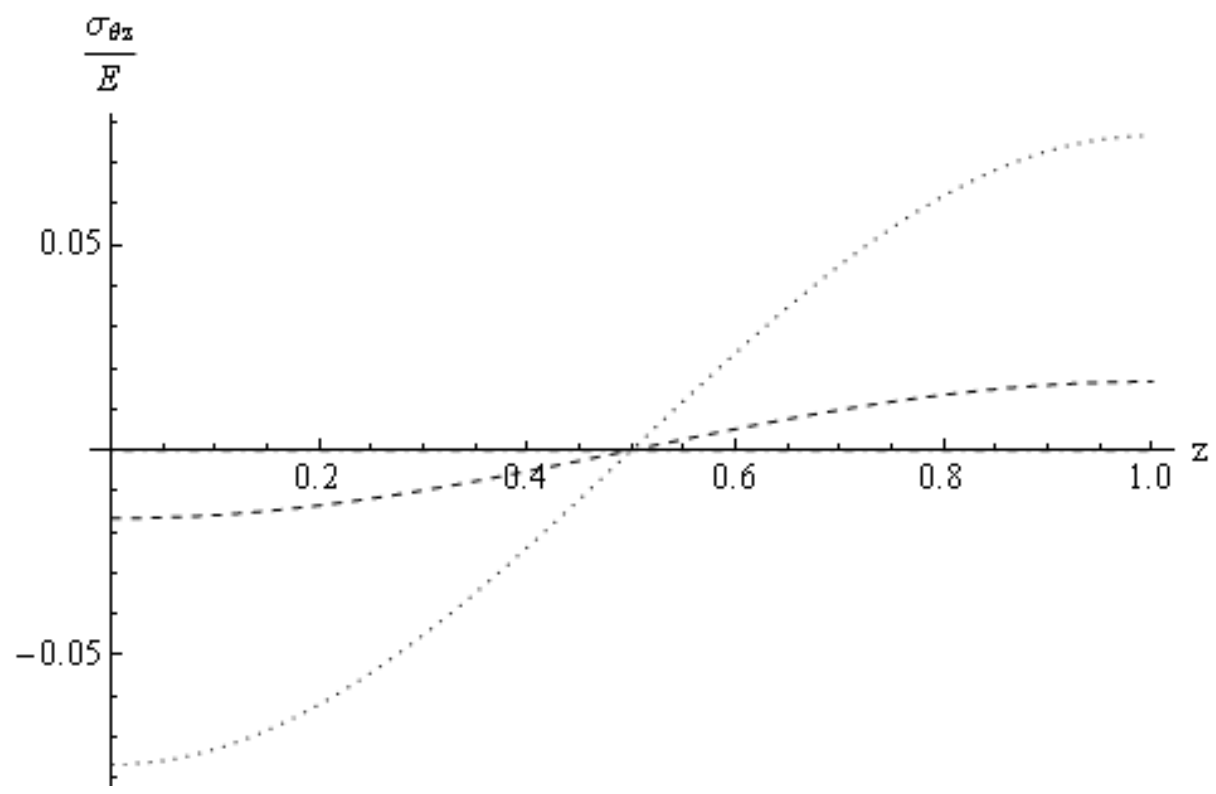

Figure 8.3: $\sigma_{\theta z}(r=a, z)$ : Truncation at $n=4---$, Timoshenko $-\cdot-$, Euler-Bernoulli ...

The Timoshenko theory generates a stress that is small compared to the other two as the stress coincides with the $z$-axis. The Euler-Bernoulli theory generates the largest stresses. Also in the case this stress is at the outer surface. 
The lateral boundary conditions that $\sigma_{r r}=\sigma_{r \theta}=\sigma_{r z} \equiv 0$ at $r=a$ are not shown here. It could be mentioned that for the current beam theory all these three stresses are zero at $r=a$ but for the classical theories this is not generally true. Hence the classical stresses do not fulfill the lateral boundary conditions.

In figure 8.4 and $8.5 \sigma_{z z}$ and $\sigma_{\theta z}$ are also plotted for a $z=3 L / 4$

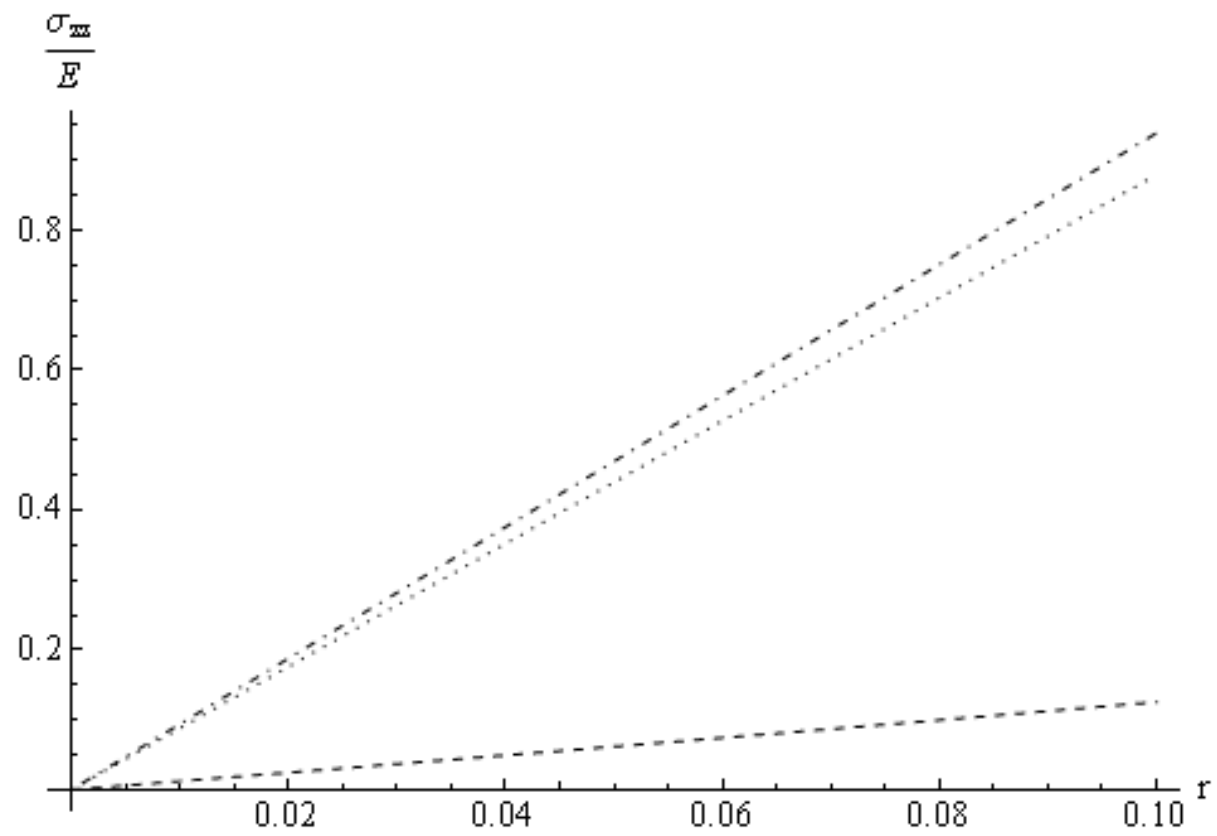

Figure 8.4: $\sigma_{z z}(r, z=3 L / 4)$ : Truncation at $n=4---$, Timoshenko $-\cdot-$, Euler-Bernoulli ...

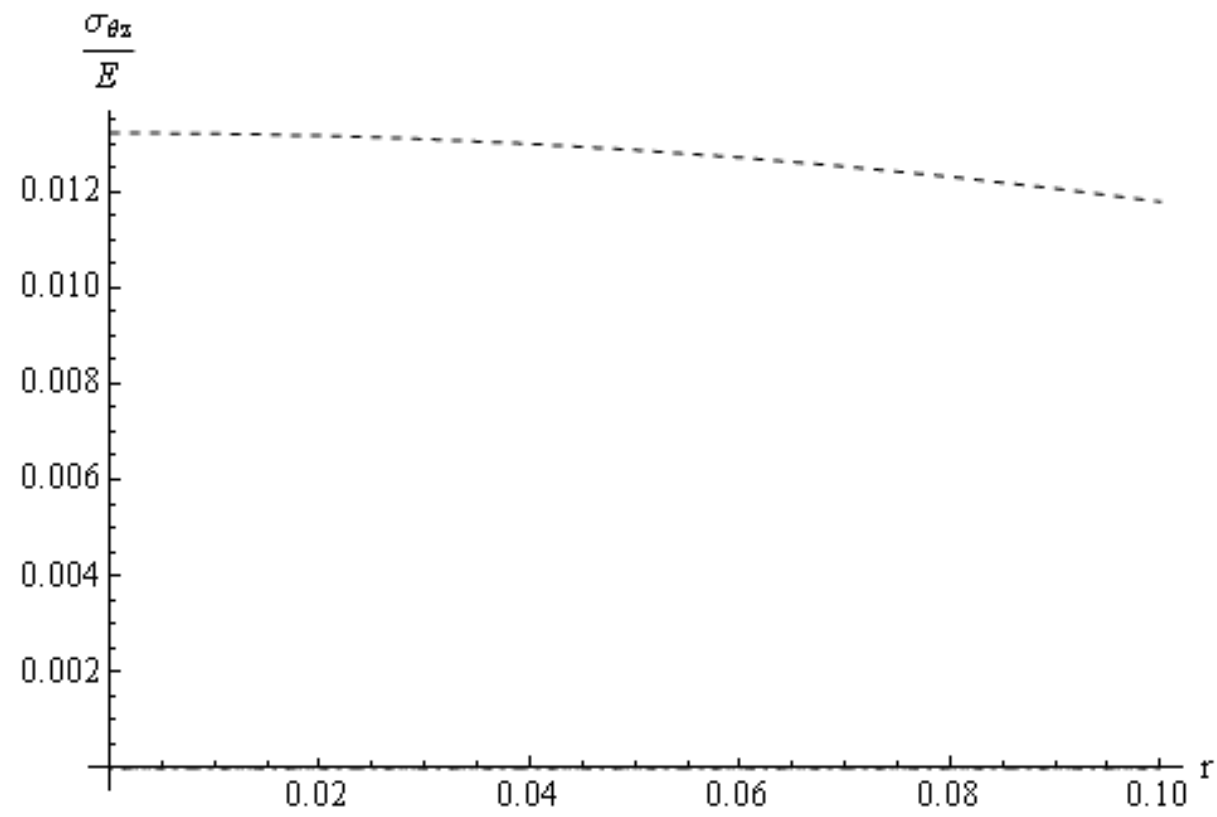

Figure 8.5: $\sigma_{\theta z}(r, z=3 L / 4)$ : Truncation at $n=4---$, Timoshenko $-\cdot-$, Euler-Bernoulli ...

As in figure 8.2 the stress $\sigma_{z z}$ is smaller for the current beam theory than the classical theories. $\sigma_{\theta z}$ is smaller for the classical theories compared to current beam theory although even for the current beam theory this shear stress is smaller than for instance $\sigma_{z z}$. In figure 8.5 the stresses produced by the classical theories coincide with the $r$-axis. 
Lastly the stress $\sigma_{r z}$ is plotted as a function $r$ for $z=3 L / 4$, in figure 8.6.

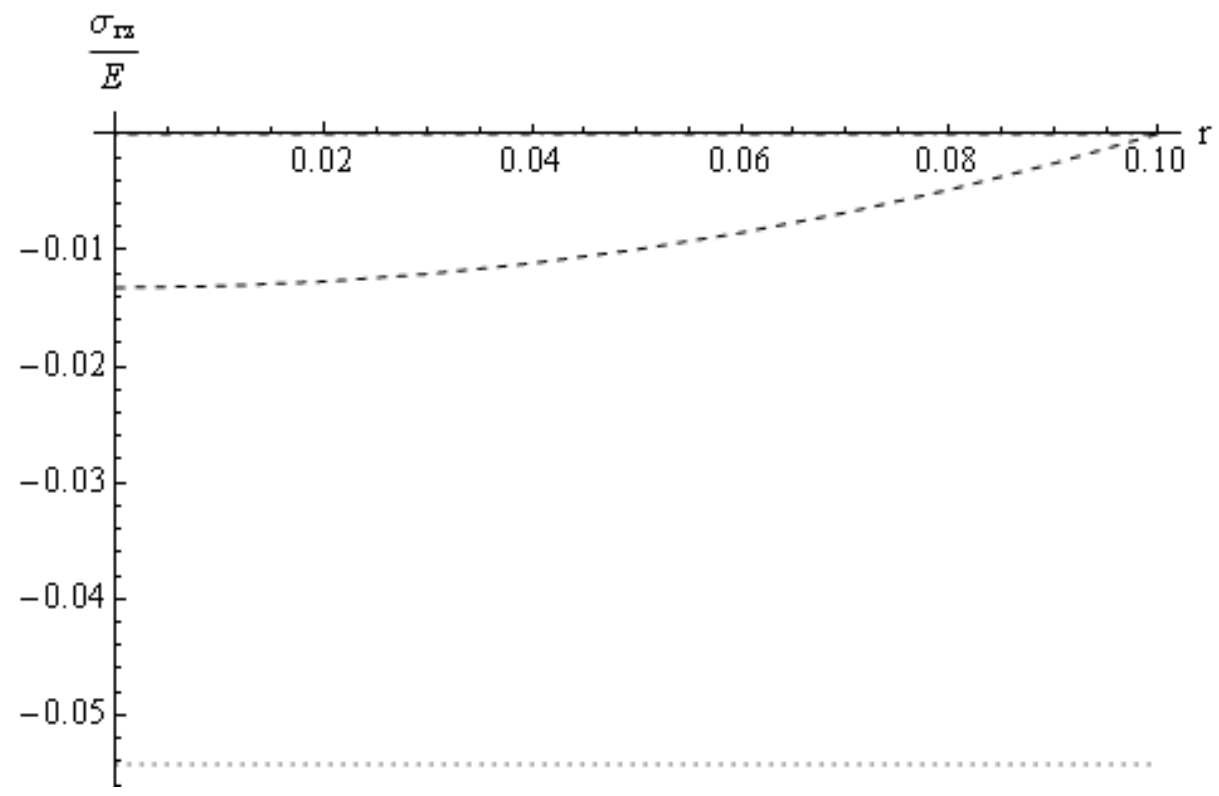

Figure 8.6: $\sigma_{r z}(r, z=3 L / 4)$ : Truncation at $n=4---$, Timoshenko - - Euler-Bernoulli ...

For this case the Timoshenko theory generates small stresses, that coincide with the $r$-axis, compared to the current beam theory and the Euler-Bernoulli theory. The EulerBernoulli theory develops a constant stress that is larger than the current beam theory. The current beam theory generates a stress that varies along the radial coordinate and it can also be seen that the stress is zero at $r=a$ as stated in the lateral boundary conditions. 


\section{Concluding Remarks}

This thesis investigates whether the method proposed by Boström [2] is applicable for obtaining governing beam equations. The obtained equations are assumed to approximate the exact theory asymptotically. This characteristic is examined by comparing dispersion curves and eigenfrequencies for a particular case of boundary conditions. It is seen that the governing equations approximate the exact theory better as more terms are accounted for and thus it is not unreasonable to assume that the method proposed by [2] generates asymptotically correct equations. Comparisons of displacement fields and stress fields are also conducted from which shows that there is a substantial difference between the theory developed in this thesis and the classical beam theories. Finally it is concluded that the presented method gives satisfying results for studying beams. 


\section{References}

[1] J.D. Achenbach, Wave Propagation in Elastic Solids, North-Holland, 1973.

[2] A. Boström, On wave equations for elastic rods, Z. Angew. Math. Mech. 80 (2000) 245-251.

[3] P.D. Folkow and K. Mauritsson, Dynamic higher-order equations for finite rods, Q. Jl. Mech. appl. Math. 63 (2010) 1-22.

[4] A.M. Hägglund and P.D. Folkow, Dynamic cylindrical shell equations by power series exapnsion, Int.J. Solids Struct. 45 (2008) 4509-4522.

[5] K. Mauritsson. Dynamic anisotropic and piezoelectric plate equations: a power series approach with recursion relations among the expansion functions, $\mathrm{PhD}$ thesis, Chalmers University of Technology, 2010.

[6] A. Boström, G. Johansson and P. Olsson, On the rational derivation of a hierarchy of dynamic equations for a homogeneous, isotropic, elastic plate, Int. J. Solids Struct. 38 (2001) 2487-2501.

[7] J.R. Hutchinson, Shear coefficients for Timoshenko beam theory, J. Appl. Mech. 68 (2001) 87-91.

[8] L. Råde and B. Westergren, Mathematics Handbook for Science and Engineering, Studentlitteratur, 1988. 


\section{Appendices}

\section{A Uncoupled Recursion Formulas}

The recursion formulas where $u_{m+2 k+3}$ and $v_{m+2 k+3}$ are uncoupled.

$$
\begin{aligned}
& 16 \mu(\lambda+2 \mu)(k+1)(k+2)\left[(k+1)(k+2)+(3+2 k) m+m^{2}\right] u_{m+2 k+3}= \\
& {\left[\mu(m+2 k+4)(m+2 k+2)-(\lambda+2 \mu) m^{2}\right] \rho \frac{\partial^{2} u_{m+2 k+1}}{\partial t^{2}}} \\
& -\left[\mu(m+2 k+4)(m+2 k+2)-(\lambda+2 \mu) m^{2}\right] \mu \frac{\partial^{2} u_{m+2 k+1}}{\partial z^{2}} \\
& -[(\lambda+\mu)(m+2 k+2) m-2 \mu m] \rho \frac{\partial^{2} v_{m+2 k+1}}{\partial t^{2}} \\
& +[(\lambda+\mu)(m+2 k+2) m-2 \mu m] \mu \frac{\partial^{2} v_{m+2 k+1}}{\partial z^{2}} \\
& -4 \mu(\lambda+\mu)(k+1)(m+k+1)(m+2 k+4) \frac{\partial w_{m+2 k+2}}{\partial z} \\
& 16 \mu(\lambda+2 \mu)(k+1)(k+2)\left[(k+1)(k+2)+(3+2 k) m+m^{2}\right] v_{m+2 k+3}= \\
& {\left[(\lambda+2 \mu)(m+2 k+4)(m+2 k+2)-\mu m^{2}\right] \rho \frac{\partial^{2} v_{m+2 k+1}}{\partial t^{2}}} \\
& -\left[(\lambda+2 \mu)(m+2 k+4)(m+2 k+2)-\mu m^{2}\right] \mu \frac{\partial^{2} v_{m+2 k+1}}{\partial z^{2}} \\
& +[(\lambda+\mu)(m+2 k+4) m+2 \mu m] \rho \frac{\partial^{2} u_{m+2 k+1}}{\partial t^{2}} \\
& -[(\lambda+\mu)(m+2 k+4) m+2 \mu m] \mu \frac{\partial^{2} u_{m+2 k+1}}{\partial z^{2}} \\
& +4 \mu(\lambda+\mu)(k+1)(m+k+1) m \frac{\partial w_{m+2 k+2}}{\partial z} \\
& \mu\left[(m+2 k+2)^{2}-m^{2}\right] w_{m+2 k+2}=\rho \frac{\partial^{2} w_{m+2 k}}{\partial t^{2}}-(\lambda+2 \mu) \frac{\partial^{2} w_{m+2 k}}{\partial z^{2}} \\
& -(\lambda+\mu)(m+2 k+2) \frac{\partial u_{m+2 k+1}}{\partial z}-(\lambda+\mu) m \frac{\partial v_{m+2 k+1}}{\partial z}
\end{aligned}
$$




\section{B Linear Differential Operators}

$$
\begin{aligned}
& d_{u u}=\frac{\left[\mu(m+2 k+4)(m+2 k+2)-(\lambda+2 \mu) m^{2}\right] \rho \frac{\partial^{2}}{\partial t^{2}}}{16 \mu(\lambda+2 \mu)(k+1)(k+2)\left[(k+1)(k+2)+(3+2 k) m+m^{2}\right]}- \\
& -\frac{\left[\mu(m+2 k+4)(m+2 k+2)-(\lambda+2 \mu) m^{2}\right] \mu \frac{\partial^{2}}{\partial z^{2}}}{16 \mu(\lambda+2 \mu)(k+1)(k+2)\left[(k+1)(k+2)+(3+2 k) m+m^{2}\right]}+ \\
& +\frac{4 \mu(\lambda+\mu)(k+1)(m+k+1)(m+2 k+4)}{16 \mu(\lambda+2 \mu)(k+1)(k+2)\left[(k+1)(k+2)+(3+2 k) m+m^{2}\right]} \frac{(\lambda+\mu)(m+2 k+2) \frac{\partial^{2}}{\partial z^{2}}}{\mu\left[(m+2 k+2)^{2}+m^{2}\right]} \\
& d_{u v}=-\frac{[(\lambda+\mu)(m+2 k+2) m-2 \mu m] \rho \frac{\partial^{2}}{\partial t^{2}}}{16 \mu(\lambda+2 \mu)(k+1)(k+2)\left[(k+1)(k+2)+(3+2 k) m+m^{2}\right]}+ \\
& +\frac{[(\lambda+\mu)(m+2 k+2) m-2 \mu m] \mu \frac{\partial^{2}}{\partial z^{2}}}{16 \mu(\lambda+2 \mu)(k+1)(k+2)\left[(k+1)(k+2)+(3+2 k) m+m^{2}\right]}+ \\
& +\frac{4 \mu(\lambda+\mu)(k+1)(m+k+1)(m+2 k+4)}{16 \mu(\lambda+2 \mu)(k+1)(k+2)\left[(k+1)(k+2)+(3+2 k) m+m^{2}\right]} \frac{(\lambda+\mu) m \frac{\partial^{2}}{\partial z^{2}}}{\mu\left[(m+2 k+2)^{2}+m^{2}\right]} \\
& d_{u w}=-\frac{4 \mu(\lambda+\mu)(k+1)(m+k+1)(m+2 k+4)}{16 \mu(\lambda+2 \mu)(k+1)(k+2)\left[(k+1)(k+2)+(3+2 k) m+m^{2}\right]} \frac{\rho \frac{\partial^{3}}{\partial z \partial t^{2}}}{\mu\left[(m+2 k+2)^{2}+m^{2}\right]}+ \\
& +\frac{4 \mu(\lambda+\mu)(k+1)(m+k+1)(m+2 k+4)}{16 \mu(\lambda+2 \mu)(k+1)(k+2)\left[(k+1)(k+2)+(3+2 k) m+m^{2}\right]} \frac{(\lambda+2 \mu) \frac{\partial^{3}}{\partial z^{3}}}{\mu\left[(m+2 k+2)^{2}+m^{2}\right]} \\
& d_{v u}=\frac{[(\lambda+\mu)(m+2 k+4) m+2 \mu m] \rho \frac{\partial^{2}}{\partial t^{2}}}{16 \mu(\lambda+2 \mu)(k+1)(k+2)\left[(k+1)(k+2)+(3+2 k) m+m^{2}\right]}- \\
& -\frac{[(\lambda+\mu)(m+2 k+4) m+2 \mu m] \mu \frac{\partial^{2}}{\partial z^{2}}}{16 \mu(\lambda+2 \mu)(k+1)(k+2)\left[(k+1)(k+2)+(3+2 k) m+m^{2}\right]}- \\
& -\frac{4 \mu(\lambda+\mu)(k+1)(m+k+1) m}{16 \mu(\lambda+2 \mu)(k+1)(k+2)\left[(k+1)(k+2)+(3+2 k) m+m^{2}\right]} \frac{(\lambda+\mu)(m+2 k+2) \frac{\partial^{2}}{\partial z^{2}}}{\mu\left[(m+2 k+2)^{2}+m^{2}\right]}
\end{aligned}
$$




$$
\begin{aligned}
& d_{v v}=\frac{\left[(\lambda+2 \mu)(m+2 k+4)(m+2 k+2)-\mu m^{2}\right] \rho \frac{\partial^{2}}{\partial t^{2}}}{16 \mu(\lambda+2 \mu)(k+1)(k+2)\left[(k+1)(k+2)+(3+2 k) m+m^{2}\right]}- \\
& -\frac{\left[(\lambda+2 \mu)(m+2 k+4)(m+2 k+2)-\mu m^{2}\right] \mu \frac{\partial^{2}}{\partial z^{2}}}{16 \mu(\lambda+2 \mu)(k+1)(k+2)\left[(k+1)(k+2)+(3+2 k) m+m^{2}\right]}- \\
& -\frac{4 \mu(\lambda+\mu)(k+1)(m+k+1) m}{16 \mu(\lambda+2 \mu)(k+1)(k+2)\left[(k+1)(k+2)+(3+2 k) m+m^{2}\right]} \frac{(\lambda+\mu) m) \frac{\partial^{2}}{\partial z^{2}}}{\mu\left[(m+2 k+2)^{2}+m^{2}\right]} \\
& d_{v w}=\frac{4 \mu(\lambda+\mu)(k+1)(m+k+1) m}{16 \mu(\lambda+2 \mu)(k+1)(k+2)\left[(k+1)(k+2)+(3+2 k) m+m^{2}\right]} \frac{\rho \frac{\partial^{3}}{\partial z \partial t^{2}}}{\mu\left[(m+2 k+2)^{2}+m^{2}\right]}- \\
& -\frac{4 \mu(\lambda+\mu)(k+1)(m+k+1) m}{16 \mu(\lambda+2 \mu)(k+1)(k+2)\left[(k+1)(k+2)+(3+2 k) m+m^{2}\right]} \frac{(\lambda+2 \mu) \frac{\partial^{3}}{\partial z^{3}}}{\mu\left[(m+2 k+2)^{2}+m^{2}\right]} \\
& d_{w u}=-\frac{(\lambda+\mu)(m+2 k+2)}{\mu\left[(m+2 k+2)^{2}-m^{2}\right]} \frac{\partial}{\partial z} \\
& d_{w v}=-\frac{(\lambda+\mu) m}{\mu\left[(m+2 k+2)^{2}-m^{2}\right]} \frac{\partial}{\partial z} \\
& d_{w w}=\frac{\rho}{\mu\left[(m+2 k+2)^{2}+m^{2}\right]} \frac{\partial^{2}}{\partial t^{2}}-\frac{\partial^{2}}{\mu\left[(m+2 k+2)^{2}+m^{2}\right]} \frac{(\lambda+2 \mu)}{\partial z^{2}}
\end{aligned}
$$




\section{System of Beam Equations for $n=1$}

$$
\begin{aligned}
& ((\lambda+2 \mu) m-2 \mu) u_{m-1}+\lambda m v_{m-1}+\left[((\lambda+2 \mu)(m+2)-2 \mu) u_{m+1}+\lambda m v_{m+1}+\lambda \frac{\partial w_{m}}{\partial z}\right] a^{2}+ \\
& +\left[((\lambda+2 \mu)(m+4)-2 \mu) u_{m+3}+\lambda m v_{m+3}+\lambda \frac{\partial w_{m+2}}{\partial z}\right] a^{4}=0 \\
& (m-2) v_{m-1}-m u_{m-1}+\left[m v_{m+1}-m u_{m+1}\right] a^{2}+\left[(m+2) v_{m+3}-m u_{m+3}\right] a^{4}=0 \\
& \frac{\partial u_{m-1}}{\partial z}+m w_{m}+\left[\frac{\partial u_{m+1}}{\partial z}+(m+2) w_{m+2}\right] a^{2}=0
\end{aligned}
$$

